\title{
Front Matter: Volume 8151
}

, "Front Matter: Volume 8151," Proc. SPIE 8151, Techniques and Instrumentation for Detection of Exoplanets V, 815101 (23 September 2011); doi: $10.1117 / 12.913633$

SPIE Event: SPIE Optical Engineering + Applications, 2011, San Diego, California, SPIE. United States 


\section{PROCEEDINGS OF SPIE}

\section{Techniques and Instrumentation for Detection of Exoplanets $V$}

\section{Stuart Shaklan}

Editor

23-24 August 2011

San Diego, California, United States

Sponsored and Published by

SPIE 
The papers included in this volume were part of the technical conference cited on the cover and title page. Papers were selected and subject to review by the editors and conference program committee. Some conference presentations may not be available for publication. The papers published in these proceedings reflect the work and thoughts of the authors and are published herein as submitted. The publisher is not responsible for the validity of the information or for any outcomes resulting from reliance thereon.

Please use the following format to cite material from this book:

Author(s), "Title of Paper," in Techniques and Instrumentation for Detection of Exoplanets $V$, edited by Stuart Shaklan, Proceedings of SPIE Vol. 8151 (SPIE, Bellingham, WA, 2011) Article CID Number.

ISSN 0277-786X

ISBN 9780819487612

Published by

SPIE

P.O. Box 10, Bellingham, Washington 98227-0010 USA

Telephone +1 3606763290 (Pacific Time) · Fax +1 3606471445

SPIE.org

Copyright () 2011, Society of Photo-Optical Instrumentation Engineers

Copying of material in this book for internal or personal use, or for the internal or personal use of specific clients, beyond the fair use provisions granted by the U.S. Copyright Law is authorized by SPIE subject to payment of copying fees. The Transactional Reporting Service base fee for this volume is $\$ 18.00$ per article (or portion thereof), which should be paid directly to the Copyright Clearance Center (CCC), 222 Rosewood Drive, Danvers, MA 01923. Payment may also be made electronically through CCC Online at copyright.com. Other copying for republication, resale, advertising or promotion, or any form of systematic or multiple reproduction of any material in this book is prohibited except with permission in writing from the publisher. The CCC fee code is 0277-786X/11/ \$18.00.

Printed in the United States of America.

Publication of record for individual papers is online in the SPIE Digital Library.

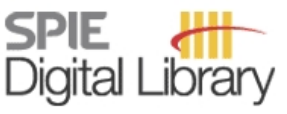

SPIEDigitalLibrary.org

Paper Numbering: Proceedings of SPIE follow an e-First publication model, with papers published first online and then in print and on CD-ROM. Papers are published as they are submitted and meet publication criteria. A unique, consistent, permanent citation identifier (CID) number is assigned to each article at the time of the first publication. Utilization of CIDs allows articles to be fully citable as soon as they are published online, and connects the same identifier to all online, print, and electronic versions of the publication. SPIE uses a six-digit CID article numbering system in which:

- The first four digits correspond to the SPIE volume number.

- The last two digits indicate publication order within the volume using a Base 36 numbering system employing both numerals and letters. These two-number sets start with 00, 01, 02, 03, 04 , $05,06,07,08,09,0 A, 0 B \ldots$. OZ, followed by 10-1Z, 20-2Z, etc.

The CID number appears on each page of the manuscript. The complete citation is used on the first page, and an abbreviated version on subsequent pages. Numbers in the index correspond to the last two digits of the six-digit CID number. 


\section{Contents}

xi

xiii

$X X V$

SESSION 1

815102

Laboratory demonstration of high-contrast imaging at inner working angles $2 \lambda / D$ and better [8151-01]

R. Belikov, E. Pluzhnik, F. C. Witteborn, T. P. Greene, D. H. Lynch, P. T. Zell, NASA Ames Research Ctr. (United States); O. Guyon, The Univ. of Arizona (United States)

815103 Influence of surface errors on the design of PIAA mirrors using numerical and semi-analytical propagation models [8151-02]

A. Carlotti, Princeton Univ. (United States); L. Pueyo, The Johns Hopkins Univ. (United States) and Space Telescope Science Institute (United States)

815104 Laboratory testing of a Phase-Induced Amplitude Apodization (PIAA) coronagraph [8151-03]

B. Kern, Jet Propulsion Lab. (United States); O. Guyon, Steward Observatory, The Univ. of Arizona (United States); A. Give'on, A. Kuhnert, A. Niessner, Jet Propulsion Lab. (United States)

815105 Diamond turned high precision PIAA optics and four mirror PIAA system for high contrast imaging of exo-planets [8151-04]

K. Balasubramanian, E. Cady, Jet Propulsion Lab. (United States); L. Pueyo, The Johns Hopkins Univ. (United States); X. An, S. Shaklan, Jet Propulsion Lab. (United States); O. Guyon, Steward Observatory, The Univ. of Arizona (United States) and Subaru Telescope, National Astronomical Observatory of Japan (United States); R. Belikov, NASA Ames Research Ctr. (United States)

815106 Studies of the effects of optical system errors on the HCIT contrast performance [8151-05] E. Sidick, S. Shaklan, A. Give'on, B. Kern, Jet Propulsion Lab. (United States) 
815108 Taking the vector vortex coronagraph to the next level for ground-and space-based exoplanet imaging instruments: review of technology developments in the USA, Japan, and Europe (Invited Paper) [8151-07]

D. Mawet, European Southern Observatory (Chile) and Jet Propulsion Lab. (United States); N. Murakami, Hokkaido Univ. (Japan); C. Delacroix, Univ. de Liège (Belgium); E. Serabyn, Jet Propulsion Lab. (United States); O. Absil, Univ. de Liège (Belgium); N. Baba, Hokkaido Univ. (Japan); J. Baudrand, A. Boccaletti, Observatoire de Paris à Meudon (France); R. Burruss, Jet Propulsion Lab. (United States); R. Chipman, College of Optical Sciences, The Univ. of Arizona (United States); P. Forsberg, Uppsala Univ. (Sweden); S. Habraken, Univ. de Liège (Belgium); S. Hamaguchi, Hokkaido Univ. (Japan); C. Hanot, Univ. de Liège (Belgium); A. Ise, Hokkaido Univ. (Japan); M. Karlsson, Uppsala Univ. (Sweden); B. Kern, J. Krist, A. Kuhnert, M. Levine, K. Liewer, Jet Propulsion Lab. (United States); S. McClain, College of Optical Sciences, The Univ. of Arizona (United States); S. McEldowney, Microsoft (United States); B. Mennesson, D. Moody, Jet Propulsion Lab. (United States); H. Murakami, Japan Aerospace Exploration Agency (Japan); A. Niessner, Jet Propulsion Lab. (United States); J. Nishikawa, National Astronomical Observatory of Japan (Japan); N. O'Brien, JDSU (United States); K. Oka, Hokkaido Univ. (Japan); P. Park, Jet Propulsion Lab. (United States); P. Piron, Univ. de Liège (Belgium); L. Pueyo, Space Telescope Science Institute (United States); P. Riaud, Univ. de Liège (Belgium); M. Sakamoto, Hokkaido Univ. (Japan); M. Tamura, National Astronomical Observatory of Japan (Japan); J. Trauger, Jet Propulsion Lab. (United States); D. Shemo, JDSU (United States); J. Surdej, Univ. de Liège (Belgium); N. Tabirian, BEAM Engineering for Advanced Measurements Co. (United States); W. Traub, J. Wallace, Jet Propulsion Lab. (United States); K. Yokochi, Tokyo Univ. of Agriculture and Technology (Japan)

815109 Stability error budget for an aggressive coronagraph on a $3.8 \mathrm{~m}$ telescope [8151-08] S. B. Shaklan, L. Marchen, J. Krist, M. Rud, Jet Propulsion Lab. (United States)

$8151 \mathrm{OA}$ Imaging power of multi-fibered nulling telescopes for extra-solar planet characterization [8151-09]

F. Hénault, CNRS, Univ. de Nice Sophia Antipolis, Observatoire de la Côte d'Azur (France)

$8151 \mathrm{OB}$ Current results of the PERSEE testbench: the cophasing control and the polychromatic null rate [8151-10]

J. Lozi, Ctr. National d'Études Spatiales (France) and ONERA (France) and Partenariat Haute resolution Angulaire Sol Espace (France); F. Cassaing, ONERA (France) and Partenariat Haute resolution Angulaire Sol Espace (France); J.-M. Le Duigou, Ctr. National d'Études Spatiales (France); B. Sorrente, J. Montri, ONERA (France) and Partenariat Haute resolution Angulaire Sol Espace (France); J. Reess, E. Lhome, T. Buey, Observatoire de Paris à Meudon (France) and Partenariat Haute resolution Angulaire Sol Espace (France); F. Hénault, A. Marcotto, P. Girard, Observatoire de la Côte d'Azur (France); M. Barillot, Thales Alenia Space (France); M. Ollivier, Institut d'Astrophysique Spatiale (France); V. Coudé du Foresto, Observatoire de Paris à Meudon (France) and Partenariat Haute resolution Angulaire Sol Espace (France)

$81510 \mathrm{C}$ The possibility of application of polarization-holographic elements for the discovery and characterization of exoplanets [8151-11]

G. Kakauridze, B. Kilosanidze, Institute of Cybernetics of the Georgian Technical Univ. (Georgia) 
8151 OD TPF-Interferometer: a decade of development in exoplanet detection technology (Invited Paper) [8151-12]

S. Martin, A. Ksendzov, O. Lay, R. D. Peters, D. P. Scharf, Jet Propulsion Lab. (United States)

$8151 \mathrm{OE}$ Assessing the performance limits of internal coronagraphs through end-to-end modeling: a NASA TDEM study [8151-13]

J. E. Krist, Jet Propulsion Lab. (United States); R. Belikov, NASA Ames Research Ctr. (United

States); L. Pueyo, The Johns Hopkins Univ. (United States); D. P. Mawet, D. Moody,

J. T. Trauger, S. B. Shaklan, Jet Propulsion Lab. (United States)

8151 OF Vacuum nuller testbed (VNT) performance, characterization and null control: progress report [8151-14]

R. G. Lyon, M. Clampin, P. Petrone, U. Mallik, T. Madison, M. R. Bolcar, NASA Goddard Space Flight Ctr. (United States); M. C. Noecker, S. Kendrick, Ball Aerospace \& Technologies Corp. (United States); M. Helmbrecht, Iris-AO (United States)

$81510 G$ A hybrid Lyot coronagraph for the direct imaging and spectroscopy of exoplanet systems: recent results and prospects [8151-15]

J. Trauger, D. Moody, B. Gordon, J. Krist, Jet Propulsion Lab. (United States); D. Mawet, European Southern Observatory (Chile)

$8151 \mathrm{OH}$ Phase-Induced Amplitude Apodization (PIAA) coronagraphy: recent results and future prospects [8151-16]

O. Guyon, Steward Observatory, The Univ. of Arizona (United States) and National Astronomical Observatory of Japan (United States); B. Kern, Jet Propulsion Lab. (United States); R. Belikov, NASA Ames Research Ctr. (United States); S. Shaklan, A. Kuhnert, A. Give'on, Jet Propulsion Lab. (United States)

8151 0l Advanced speckle sensing for internal coronagraphs [8151-17]

C. Noecker, Ball Aerospace \& Technologies Corp. (United States); S. Shaklan, J. K. Wallace, B. Kern, A. Give'on, Jet Propulsion Lab. (United States); J. Kasdin, Princeton Univ. (United States); R. Belikov, NASA Ames Research Ctr. (United States); S. Kendrick, Ball Aerospace \& Technologies Corp. (United States)

8151 0J Advancing technology for starlight suppression via an external occulter [8151-18] N. J. Kasdin, D. N. Spergel, R. J. Vanderbei, Princeton Univ. (United States); D. Lisman, S. Shaklan, M. Thomson, P. Walkemeyer, V. Bach, E. Oakes, E. Cady, S. Martin, L. Marchen, Jet Propulsion Lab. (United States); B. Macintosh, R. E. Rudd, Lawrence Livermore National Lab. (United States); J. Mikula, D. Lynch, NASA Ames Research Ctr. (United States)

8151 OK A photon-counting detector for exoplanet missions [8151-19]

D. F. Figer, J. Lee, B. J. Hanold, Rochester Institute of Technology (United States); B. F. Aull, J. A. Gregory, D. R. Schuette, MIT/Lincoln Lab. (United States) 


\section{SESSION $3 \quad$ MICROLENSING}

$8151 \mathrm{OL}$ The exoplanet microlensing survey by the proposed WFIRST Observatory [8151-20]

R. Barry, J. Kruk, NASA Goddard Space Flight Ctr. (United States); J. Anderson, Space Telescope Science Institute (United States); J.-P. Beaulieu, Institut d'Astrophysique de Paris (France); D. P. Bennett, Univ. of Notre Dame (United States); J. Catanzarite, Jet Propulsion Lab. (United States); E. Cheng, Conceptual Analytics (United States); S. Gaudi, Ohio State Univ. (United States); N. Gehrels, NASA Goddard Space Flight Ctr. (United States); S. Kane, California Institute of Technology (United States); J. Lunine, Cornell Univ. (United States); T. Sumi, Osaka Univ. (Japan); A. Tanner, Mississippi State Univ. (United States); W. Traub, Jet Propulsion Lab. (United States)

\section{SESSION 4 GROUND BASED DETECTION AND CHARACTERIZATION}

8151 OM The performance of the SPHERE sub-systems in the integration lab [8151-21] F. Wildi, Observatoire de Genève (Switzerland); J.-L. Beuzit, Lab. d'Astrophysique de I'Observatoire de Grenoble (France); M. Feldt, Max-Planck-Institut für Astronomie (Germany); D. Mouillet, Lab. d'Astrophysique de l'Observatoire de Grenoble (France); K. Dohlen, Observatoire Astronomique de Marseille-Provence (France); P. Puget, Lab. d'Astrophysique de l'Observatoire de Grenoble (France); A. Baruffolo, Osservatorio Astronomico di Padova (Italy); J. Charton, Lab. d'Astrophysique de l'Observatoire de Grenoble (France); A. Bocaletti, Lab. d'Etudes Spatiales et d'Instrumentation en Astrophysique (France); R. Claudi, Osservatorio Astronomico di Padova (Italy); A. Costille, P. Feautrier, Lab. d'Astrophysique de l'Observatoire de Grenoble (France); T. Fusco, ONERA (France); R. Gratton, Osservatorio Astronomico di Padova (Italy); M. Kasper, European Southern Observatory (Germany); M. Langlois, Ctr. de Recherche Astronomique de Lyon (France); P. Martinez, Lab. d'Astrophysique de l'Observatoire de Grenoble (France); D. Mesa, Osservatorio Astronomico di Padova (Italy); D. Le Mignant, Observatoire Astronomique de Marseille-Provence (France); A. Pavlov, Max-Planck-Institut für Astronomie (Germany); C. Petit, ONERA (France); J. Pragt, ASTRON (Netherlands); P. Rabou, S. Rochat, Lab. d'Astrophysique de l'Observatoire de Grenoble (France); R. Roelfsema, ASTRON (Netherlands); J.-F. Sauvage, ONERA (France); H.-M. Schmid, ETH Zurich (Switzerland); E. Stadler, Lab. d'Astrophysique de l'Observatoire de Grenoble (France); C. Moutou, Observatoire de Genève (France)

8151 ON The ZIMPOL high contrast imaging polarimeter for SPHERE: sub-system test results [8151-22] R. Roelfsema, NOVA-ASTRON (Netherlands); D. Gisler, ETH Zurich (Switzerland); J. Pragt, NOVA-ASTRON (Netherlands); H. M. Schmid, A. Bazzon, ETH Zurich (Switzerland); C. Dominik, Univ. van Amsterdam (Netherlands); A. Baruffolo, INAF, Osservatorio Astronomico di Padova (Italy); J.-L. Beuzit, J. Charton, Univ. Joseph Fourier (France); K. Dohlen, LAM, Univ. de Provence (France); M. Downing, European Southern Observatory (Germany); E. Elswijk, NOVA-ASTRON (Netherlands); M. Feldt, Max-Planck-Institut für Astronomie (Germany); M. de Haan, NOVA-ASTRON (Netherlands); N. Hubin, M. Kasper, European Southern Observatory (Germany); C. Keller, Sterrekundig Instituut Utrecht (Netherlands); J.-L. Lizon, European Southern Observatory (Germany); D. Mouillet, Univ. Joseph Fourier (France); A. Pavlov, Max-Planck-Institut für Astronomie (Germany); P. Puget, S. Rochat, Univ. Joseph Fourier (France); B. Salasnich, INAF, Osservatorio Astronomico di Padova (Italy); P. Steiner, ETH Zurich (Switzerland); C. Thalmann, R. Waters, Astronomical Institute Anton Pannekoek (Netherlands); F. Wildi, Observatoire Astronomique de l'Univ. de Geneve (Switzerland) 
$81510 Q \quad$ The Subaru coronagraphic extreme AO project: progress report [8151-25]

F. Martinache, Subaru Telescope (United States); O. Guyon, Subaru Telescope (United States) and Univ. of Arizona (United States); V. Garrel, C. Clergeon, Subaru Telescope (United States) and Observatoire de Paris-Meudon (France); T. Groff, Princeton Univ. (United States); P. Stewart, The Univ. of Sydney (Australia); R. Russell, Subaru Telescope (United States); C. Blain, Univ. of Victoria (Canada)

8151 OR The Subaru coronagraphic extreme AO (SCExAO) system: fast visible imager [8151-26] V. Garrel, Subaru Telescope (United States) and LESIA (France); O. Guyon, Subaru Telescope (United States); P. Baudoz, LESIA (France); F. Martinache, Subaru Telescope (United States); P. Stewart, The Univ. of Sydney (Australia); J. Lozi, ONERA (France); T. Groff, Princeton Univ. (United States)

\section{SESSION 5 ASTROMETRY}

8151 OS Diffractive pupil telescope for high precision space astrometry [8151-27]

O. Guyon, Steward Observatory, The Univ. of Arizona (United States) and Subaru Telescope, National Astronomical Observatory of Japan (United States); E. Bendek, M. Ammons, Steward Observatory, The Univ. of Arizona (United States); M. Shao, S. Shaklan, Jet Propulsion Lab. (United States); R. A. Woodruff, R. Belikov, NASA Ames Research Ctr. (United States)

8151 OT Microarcsecond relative astrometry from the ground with a diffractive pupil [8151-28] S. M. Ammons, Lawrence Livermore National Lab. (United States); E. A. Bendek, Steward Observatory, The Univ. of Arizona (United States); O. Guyon, Steward Observatory, The Univ. of Arizona (United States) and Subaru Telescope (United States)

8151 OU Dynamic distortion calibration using a diffracting pupil: high precision astrometry laboratory demonstration for exoplanet detection [8151-29]

E. A. Bendek, Steward Observatory, The Univ. of Arizona (United States); S. M. Ammons, Lawrence Livermore National Lab. (United States); H. Shankar, O. Guyon, Steward Observatory, The Univ. of Arizona (United States)

8151 OV NEAT: a microarcsec astrometric telescope [8151-30]

M. Shao, B. Nemati, C. Zhai, R. Goullioud, Jet Propulsion Lab. (United States); F. Malbet, IPAG (France); A. Leger, Univ. Paris (France)

8151 oW Micro-pixel image position sensing testbed [8151-31]

B. Nemati, M. Shao, C. Zhai, H. Erlig, R. Goullioud, X. Wang, Jet Propulsion Lab. (United States)

\section{SESSION 6 WAVEFRONT ESTIMATION AND CONTROL}

$81510 X$ Designing an optimal estimator for more efficient wavefront correction [8151-32]

T. D. Groff, N. J. Kasdin, Princeton Univ. (United States)

8151 OY Unified coronagraph and wavefront control design [8151-33]

N. J. Kasdin, A. Carlotti, Princeton Univ. (United States); L. Pueyo, Space Telescope Science Institute (United States); T. Groff, R. Vanderbei, Princeton Univ. (United States) 
$81510 Z$ Progress on broadband control and deformable mirror tolerances in a 2-DM system [8151-34]

T. D. Groff, A. Carlotti, N. J. Kasdin, Princeton Univ. (United States)

815110 Pair-wise, deformable mirror, image plane-based diversity electric field estimation for high contrast coronagraphy [8151-35]

A. Give'on, B. D. Kern, S. Shaklan, Jet Propulsion Lab. (United States)

$815111 \quad$ Kernel-phases for high-contrast detection beyond the resolution limit [8151-36]

F. Martinache, Subaru Telescope (United States)

\section{SESSION 7 STARSHADES}

815112 Nondimensional representations for occulter design and performance evaluation [8151-37] E. Cady, Jet Propulsion Lab. (United States)

815113 A starshade petal error budget for exo-earth detection and characterization [8151-38] S. B. Shaklan, L. Marchen, P. D. Lisman, E. Cady, S. Martin, M. Thomson, Jet Propulsion Lab. (United States); P. Dumont, KinetX Inc. (United States); N. J. Kasdin, Princeton Univ. (United States)

815114 Optical verification of occulter-based high contrast imaging [8151-39]

D. Sirbu, Princeton Univ. (United States); E. J. Cady, Jet Propulsion Lab. (United States);

N. J. Kasdin, R. J. Vanderbei, J. Lu, E. Kao, Princeton Univ. (United States)

\section{POSTER SESSION}

815115 Higher-precision radial velocity measurements with the SOPHIE spectrograph using octagonal-section fibers [8151-40]

S. Perruchot, CNRS, Observatoire de Haute-Provence (France); F. Bouchy, CNRS, Institut d'Astrophysique de Paris (France) and CNRS, Observatoire de Haute-Provence (France); B. Chazelas, Observatoire de Genève (Switzerland); R. F. Díaz, G. Hébrard, CNRS, Institu† d'Astrophysique de Paris (France) and CNRS, Observatoire de Haute-Provence (France); K. Arnaud, CNRS, Ctr. de Physique des Particules de Marseille (France); L. Arnold, CNRS, Observatoire de Haute-Provence (France); G. Avila, European Southern Observatory (Germany); X. Delfosse, CNRS, IPAG (France); I. Boisse, CNRS, Institut d'Astrophysique de Paris (France); G. Moreaux, CNRS, Lab. d'Astrophysique de Marseille (France); F. Pepe, CNRS, Ctr. de Physique des Particules de Marseille (France); Y. Richaud, CNRS, Observatoire de Haute-Provence (United States); A. Santerne, Observatoire de Genève (Switzerland); R. Sottile, CNRS, Observatoire de Haute-Provence (France); D. Tézier, Observatoire de Genève (Switzerland) 
815116 Design of a star, planet and exo-zodiacal cloud simulator for the nulling testbench PERSEE [8151-41]

F. Hénault, Univ. de Nice Sophia Antipolis (France); P. Girard, Observatoire de la Cote d'Azur (France); A. Marcotto, N. Mauclert, C. Bailet, Univ. de Nice Sophia Antipolis (France);

B. Lopez, Univ Nice-Sophia Antipolis (France); F. Millour, Y. Rabbia, A. Roussel, Univ. de Nice Sophia Antipolis (France); M. Barillot, Thales Alenia Space (France); J. Lozi, F. Cassaing, ONERA (France); K. Houairi, Thales Alenia Space (France); B. Sorrente, J. Montri, ONERA (France); E. Lhome, Observatoire de Paris (France); J.-M. Reess, L. Pham, J. Buey, V. Coudé du Foresto, S. Jacquinod, Observatoire de Paris à Meudon (France); M. Ollivier, IAS (France); J.-M. Le Duigou, Ctr. National d'Études Spatiales (France)

815117 DEBRIS sightings in the Kepler field [8151-42]

F. C. Witteborn, J. Van Cleve, W. Borucki, NASA Ames Research Ctr. (United States);

V. Argabright, Ball Aerospace \& Technology Corp. (United States); P. Hascall, Orbital Network Engineering (United States)

815118 Relaxing the sensitivity of nuller coronagraphs to finite stellar diameters using apodization [8151-44]

A. Carlotti, Princeton Univ. (United States); C. Aime, Y. Rabbia , Univ. de Nice Sophia Antipolis (France);

815119 Pressure and temperature stabilization of an existing chelle spectrograph II [8151-45] F. Grupp, Max Planck Institute for Extraterrestrial Physics (Germany) and Univ. Observatory Munich (Germany); A. Brucalassi, Max Planck Institute for Extraterrestrial Physics (Germany): F. Lang, Univ. Observatory Munich (Germany); S. M. Hu, Shandong Univ. at Weihai (China); R. Holzwarth, T. Udem, Max-Planck-Institute for Quantum Optics (Germany); U. Hopp, Univ. Observatory Munich (Germany); R. Bender, Max Planck Institute for Extraterrestrial Physics (Germany) and Univ. Observatory Munich (Germany)

8151 1A Speckle identification to assist the direct detection of exoplanets [8151-46]

E. J. Young, N. J. Kasdin, A. Carlotti, M. Littman, Princeton Univ. (United States);

M. C. Noecker, Ball Aerospace \& Technologies Corp. (United States)

8151 1B Wide-field telescope design for the KMTNet project [8151-47]

S.-L. Kim, B.-G. Park, C.-U. Lee, Korea Astronomy and Space Science Institute (Korea, Republic of); L. Kappler, N. Kappler, TBR Construction \& Engineering (United States); W. Poteet, H. Cauthen, D. Blanco, R. Buchroeder, CP Systems, Inc. (United States); J. Teran, S. Freestone, M3 Engineering \& Technology Corp. (United States); J.-U. Lee, Cheongju Univ. (Korea, Republic of); M. Cho, National Optical Astronomy Observatory (United States); I.-S. Yuk, M.-Y. Chun, Korea Astronomy and Space Science Institute (Korea, Republic of); H. Jin, Kyung Hee Univ. (Korea, Republic of); S.-M. Cha, Korea Astronomy and Space Science Institute (Korea, Republic of)

8151 1C Achieving high-precision ground-based photometry for transiting exoplanets [8151-48] O. Guyon, Subaru Telescope, National Astronomical Observatory of Japan (United States) and Steward Observatory, The Univ. of Arizona (United States); F. Martinache, Subaru Telescope, National Astronomical Observatory of Japan (United States) 
8151 ID Recent results of the second generation of vector vortex coronagraphs on the high-contrast imaging testbed at JPL [8151-49]

D. Mawet, European Southern Observatory (Chile) and Jet Propulsion Lab. (United States);

E. Serabyn, D. Moody, B. Kern, A. Niessner, A. Kuhnert, Jet Propulsion Lab. (United States);

D. Shemo, JDSU (United States); R. Chipman, S. McClain, The Univ. of Arizona (United States); J. Trauger, Jet Propulsion Lab. (United States)

8151 IE Zodiac II: debris disk science from a balloon [8151-50]

G. Bryden, W. Traub, L. C. Roberts, Jr., R. Bruno, S. Unwin, Jet Propulsion Lab. (United States); S. Backovsky, ATK Space Systems (United States); P. Brugarolas, Jet Propulsion Lab. (United States); S. Chakrabarti, Boston Univ. (United States); P. Chen, Jet Propulsion Lab. (United States); L. Hillenbrand, California Institute of Technology (United States); J. Krist, Jet Propulsion Lab. (United States); C. Lillie, Northrop Grumman Aerospace Systems (United States); B. Macintosh, Lawrence Livermore National Lab. (United States); D. Mawet, B. Mennesson, D. Moody, Z. Rahman, Jet Propulsion Lab. (United States); J. Rey, Northrop Grumman Aerospace Systems (United States); K. Stapelfeldt, NASA Goddard Space Flight Ctr. (United States); D. Stuchlik, NASA Wallops Flight Facility (United States); J. Trauger, G. Vaischt, Jet Propulsion Lab. (United States)

$8151 \mathrm{fF}$ The performance of the new Fabry-Perot calibration system of the radial velocity spectrograph HARPS [8151-51]

F. Wildi, F. Pepe, B. Chazelas, Observatoire de Genève (Switzerland); G. Lo Curto, European Southern Observatory (Germany); C. Lovis, Observatoire de Genève (Switzerland)

8151 1G Deep UV to NIR space telescopes and exoplanet coronagraphs: a trade study on throughput, polarization, mirror coating options and requirements [8151-52]

K. Balasubramanian, S. Shaklan, A. Give'on, E. Cady, L. Marchen, Jet Propulsion Lab. (United States)

Author Index 


\title{
Conference Committee
}

\author{
Program Track Chair
}

Oswald H. Siegmund, University of California, Berkeley (United States)

Conference Chair

Stuart Shaklan, Jet Propulsion Laboratory (United States)

Program Committee

David P. Bennett, University of Notre Dame (United States)

Olivier Guyon, Subaru Telescope, National Astronomical Observatory of Japan (United States) and Research Corporation of the University of Hawaii (United States) and The University of Arizona (United States)

Richard G. Lyon, NASA Goddard Space Flight Center (United States)

Bruce A. Macintosh, Lawrence Livermore National Laboratory (United States)

M. Charley Noecker, Ball Aerospace \& Technologies Corporation (United States)

Rémi Soummer, Space Telescope Science Institute (United States)

\section{Session Chairs}

1 Coronagraph Design and Performance

Richard G. Lyon, NASA Goddard Space Flight Center (United States)

2 TDEM-1 Progress

Stuart Shaklan, Jet Propulsion Laboratory (United States)

3 Microlensing

Stuart Shaklan, Jet Propulsion Laboratory (United States)

4 Ground Based Detection and Characterization

Dimitri P. Mawet, European Southern Observatory (Chile)

5 Astrometry

N. Jeremy Kasdin, Princeton University (United States)

6 Wavefront Estimation and Control

Olivier Guyon, Subaru Telescope, National Astronomical Observatory of Japan (United States) and Research Corporation of the University of Hawaii (United States) and The University of Arizona (United States) 
$7 \quad$ Starshades

Stefan R. Martin, Jet Propulsion Laboratory (United States) 


\title{
The Little Photometer That Could: Technical Challenges and Science Results from the Kepler Mission
}

\author{
Jon M. Jenkins $^{\circ a}$, Jeb Dunnuck ${ }^{b}$, \\ ${ }^{a}$ SETI Institute/NASA Ames Research Center, M/S 244-30, Moffett Field, CA USA 94305 \\ ${ }^{b}$ Ball Aerospace \& Technologies Corporation, P.O. Box 1062, Boulder, CO, USA 80306
}

\begin{abstract}
The Kepler spacecraft launched on March 7, 2009, initiating NASA's first search for Earth-size planets orbiting Sun-like stars. Since launch, Kepler has announced the discovery of 17 exoplanets, including a system of six transiting a Sun-like star, Kepler-11, and the first confirmed rocky planet, Kepler-10b, with a radius of 1.4 that of Earth. Kepler is proving to be a cornucopia of discoveries: it has identified over 1200 candidate planets based on the first 120 days of observations, including 54 that are in or near the habitable zone of their stars, and 68 that are 1.2 Earth radii or smaller. An astounding 408 of these planetary candidates are found in 170 multiple systems, demonstrating the compactness and flatness of planetary systems composed of small planets. Never before has there been a photometer capable of reaching a precision near 20 ppm in 6.5 hours and capable of conducting nearly continuous and uninterrupted observations for months to years. In addition to exoplanets, Kepler is providing a wealth of astrophysics, and is revolutionizing the field of asteroseismology. Designing and building the Kepler photometer and the software systems that process and analyze the resulting data to make the discoveries presented a daunting set of challenges, including how to manage the large data volume. The challenges continue into flight operations, as the photometer is sensitive to its thermal environment, complicating the task of detecting 84 ppm drops in brightness corresponding to Earth-size planets transiting Sun-like stars.
\end{abstract}

Keywords: Kepler Mission, exoplanet, transit, photometry, data compression, systematic error correction

\section{INTRODUCTION}

The Kepler spacecraft was launched in March 2009 as NASA's first mission capable of finding Earth-size planets orbiting Sun-like stars. This mission has been a spectacular success in terms of revolutionizing both the fields of extrasolar planets and of asteroseismology, the study of stellar oscillations. Over 1200 planetary candidates were detected in the first 120 days of observations, ${ }^{1}$ effectively tripling the number of known extrasolar planets as of February 2011. Most of these candidates are as small or smaller than Neptune. Kepler finds multiple planet candidates transiting a star $\sim 17 \%$ of the time. With the capability to conduct observations for at least 10 years and a photometric precision approaching $20 \mathrm{ppm}$ on 6.5-hr timescales, Kepler is poised to reach its primary goal of determining the frequency and distribution of rocky planets in the habitable zone (HZ) of solar-like stars, that range of distances for which liquid water could pool on the surface of a terrestrial planet. However, the success of the Kepler Mission wasn't always a foregone conclusion.

This paper describes some of the major challenges facing Kepler both before and after launch. These challenges have been mitigated by a combination of good design, great hardware, flexible software and the talented people involved in the mission. It required collaboration between the engineers and scientists who designed and built the photometer and spacecraft and those who process and analyze the exquisite photometric data. We focus on some of the key challenges that were met handily by the design, including the performance of the onboard compression scheme and the approach to target management, and some issues that have arisen after launch when we had the first opportunity to see how a photometer such as Kepler would perform in the space environment. It's true that these one-of-a-kind machines never cease to amaze or surprise us when they are finally in orbit and operating, in part, because we cannot truly replicate the totality of the space environment in the laboratory. Also, we cannot ever anticipate the myriad complexity of nature when we observe it with a new and much more powerful instrument than has been previously available. We give examples of how we are dealing with some of the post-launch complexities in ground-processing software, including the thermal sensitivity of the photometer. We provide an update on the key scientific achievements obtained with Kepler to date. Finally, we conclude with some thoughts on what the future holds for Kepler, and how our experience with this marvelous machine can provide lessons for future space missions, such as ESA's envisioned PLATO space transit survey.

${ }^{\circ}$ Send correspondence to J.M.J.: E-mail: Jon.Jenkins@ @nasa.gov

UV/Optical/IR Space Telescopes and Instruments: Innovative Technologies and Concepts V, edited by Howard A. MacEwen, James B. Breckinridge, Proc. of SPIE Vol. 8146, 814602 - ( ) 2011 SPIE CCC code: $0277-786 \mathrm{X} / 11 / \$ 18 \cdot$ doi: $10.1117 / 12.897767$ 


\section{KEY CHALLENGES}

In 1994, the first proposal for Kepler to NASA's Discovery Program was turned down as too expensive and too risky from a technical perspective. In fact, Kepler was rejected by the next two Discovery panels in 1996 and 1998, but was ultimately selected for launch in the fourth Discovery Program competition in 2001 after several years of dedicated and intense efforts to demonstrate the technical and budgetary feasibility of the mission in the face of many significant technical challenges.

Kepler's goal is simple: to observe a large sample of solar-like stars (those burning hydrogen in their cores) to detect periodic dimming in brightness caused when a small planet transits, i.e., crosses the face of its star as it swings around in its orbit, as viewed by the spacecraft. There are several complications with this approach:

First, the dimming caused by a small planet transiting its star is very slight. Planets are rather small compared to the size of stars, even main-sequence stars, e.g., our Sun, which are called dwarfs in comparison to their older, evolved brothers, the giant stars, which burn helium in their cores. The amplitude of the dimming during transit is the ratio of the area blocked by the planet to the total area of the star. Jupiter is about $10 \%$ the radius of the Sun, so its transits block $1 \%$ of the light as viewed by an extrasolar observer. By comparison, Earth is about $10 \%$ the radius of Jupiter and consequently causes a $0.01 \%$ or 1 part in 10,000 drop in brightness when it transits the Sun. Since the opportunity to detect a signal arises only when the signal is strong compared to the observational noise, robustly detecting an Earth-size planet transiting a Sun-like star requires a signal-to-noise ratio (SNR) of at least $4 \sigma$ over the duration of a typical transit, $\sim 6.5$ hours in order to build up a total SNR $>8 \sigma$ from four or more transit events. The threshold of $7.1 \sigma$ is required in light of the large number $\left(\sim 10^{12}\right)$ of statistical tests performed to identify the transit sequences, since we don't know the period or the time of first transit. ${ }^{2,3}$ In the 1990s, the precision achieved by state of the art differential photometry from the ground with Charge Coupled Devices (CCDs) was $\sim 0.1 \%$, and could not be achieved easily on a routine basis even with $4-\mathrm{m}$ class telescopes due to atmospheric effects such as extinction, and scintillation, ${ }^{4}$ which continue to limit the precision of ground-based photometry.

The Kepler concept called for balancing the three major sources of noise to achieve a single transit SNR of $4 \sigma$ for an 84-ppm, 6.5-hr transit of a G2V star by an Earth-size planet in a one-year orbit. This point design established the three major noise sources and their required values as: 1) shot noise from Poisson photon-counting statistics $(\leq 14 \mathrm{ppm})$, instrument noise $(\leq 10 \mathrm{ppm})$, and stellar variability $(\leq 10 \mathrm{ppm}) .{ }^{5}$ A major question raised by the first three Discovery review panels was whether CCDs could achieve an instrumental precision of $10 \mathrm{ppm}$ or less, and further, whether a photometer composed of CCDs operating in a space-like environment could achieve this goal. After all, even though a telescope in deep space avoids the problems of peering up through the atmospheric soup surrounding the Earth, there are instrumental effects and noise even in the space environment. A series of laboratory measurements established that frontside illuminated CCDs can achieve the precision necessary for Kepler ${ }^{6}$ The arrival of readily available backside-thinned, illuminated CCDs brought the quantum efficiency of the proposed detectors to $>90 \%$, enhancing the prospects for Kepler, as this improvement significantly reduced the shot noise in measuring the brightness of star images. Laboratory studies with backside-illuminated CCDs demonstrated that they, too, could be operated in such a way as to achieve several ppm of instrumental noise on transit timescales, ${ }^{7}$ and that they experienced less sub-pixel variations in sensitivity. Finally, the Kepler Technology Demonstration (KTD), funded and conducted after the 1998 Discovery proposal cycle, demonstrated that the Kepler design could reach the requisite instrumental precision in the face of a variety of disturbances of importance to a space-borne photometry mission. ${ }^{8,9}$ These included residual pointing errors due to the rumble of reaction wheels used to control pointing and the band-limited performance of the attitude control system. Cosmic rays, bright, saturated stars in the field of view, and focus changes due to changing thermal conditions were also simulated. The KTD also provided direct simulations of Earth-size transit signals through the application of a small current through thin, ribbon wires placed across rectangular pinholes in the opaque plate that were imaged onto the test $\mathrm{CCD}^{*}$, an EEV $42-80^{\dagger}$. A few milliamperes of current heated the wires, causing them to expand by about one atom's width, thereby blocking $\sim 0.01 \%$ of the rectangular pinhole. The results allowed us to demonstrate that we could detect Earth-size transit signals against the shot noise and instrumental effects we knew would be at play in the space environment.

Second, the timescale and low duty cycle of transit signatures present serious challenges to Earth-bound transit photometry campaigns: a central transit of an Earth-size planet of a Sun-size star in an Earth-size orbit ( $1 \mathrm{AU})$ lasts for $\sim 13$ hours, and repeats only once per year. It is difficult to imagine achieving measurements of a quality and continuity from

\footnotetext{
${ }^{*}$ An ingenious concept due to David Koch, Deputy Science Principal Investigator.

${ }^{\dagger}$ The E2V CCDs built and flown on Kepler were derived from the EEV 42-80 CCD.
} 
the ground required to reliably observe, let alone, detect transits of an Earth-size planet, even if the requisite photometric precision could be routinely obtained. It is true that prior to Kepler's launch, $\sim 70$ planets were found that transited their parent star. Almost all of these are at least as large or larger than Jupiter, providing transit depths of $\sim 1 \%$, and in very short period orbits of only a few to several days. This set of circumstances allows for many transits during observing seasons lasting a few months, and transit durations lasting from one to a few hours, permitting detection from the ground. The fact that the stars can only be observed in visible wavelengths at night, the unpredictable nature of weather (which often interferes with astronomical observations), and the challenges in scheduling coordinated observations at multiple telescopes distributed around the globe all contribute to the difficulty of detecting habitable Earth-size planets from the ground ${ }^{\ddagger}$.

Third, the geometrical probability that the orbit of an extrasolar planet will be aligned so that we can observe its transits is small for an Earth-like orbit: the probability is the ratio of the size of the star to the size of the orbit. Thus, observers in our solar neighborhood have a $0.5 \%$ chance of seeing the Earth transit the Sun. This implies that for a robust null result, Kepler needs to observe thousands of stars in order to place strong upper limits on the frequency of such planets in the case that none are detected.

Fourth, we don't know when a star is going to dim due to a transit, and transits of terrestrial bodies transpire on timescales of 1-16 hours. The duty cycle of a transiting Earth-analog is only $0.15 \%$. Kepler counts on building the SNR of a transit signature by combining together separate events and will be observing a handful of transits for Earth-analogs.

The solution to these challenges is to launch a photometer with a sufficiently sized aperture into a heliocentric, Earthtrailing orbit with a field of view large enough to observe a sample of $\geq 100,000$ solar-like stars with instrumental "Kepler" magnitudes, $K p$ from 9 to 15 as continuously and contiguously as possible. ${ }^{5,10}$ The design and build-up of the focal plane array, the largest ever built and launched by NASA, and the photometer itself were extremely challenging endeavors in the cost- and schedule-constrained aegis of NASA's Discovery Program. ${ }^{11,12}$ Kepler's Earth-trailing orbit provides a quiescent and stable environment in which to operate. The spacecraft's thermal environment is largely controlled by the measured and stately progress of the apparent position of the Sun moving along the ecliptic from Kepler's point of view. The greatest force on the spacecraft is the torque due to the force of the photon pressure on the spacecraft and instrument. The Schmidt design of the photometer, a telescope with a $0.95-\mathrm{m}$ aperture and a $1.4-\mathrm{m}$ primary mirror, coupled with a focal plane that consists of 42 CCDs containing 94,617,600 pixels spanning 115 square degrees of sky, allow Kepler to simultaneously and nearly continuously observe $\sim 165,000$ stars. The target stars were selected using the Kepler Input Catalog (KIC) to identify the stars for which we can most readily detect and confirm transits of small rocky planets. ${ }^{13,14}$ The Attitude Determination and Control System (ADCS) provides extremely precise pointing with a required and delivered performance of $r m s$ pointing errors at the level of 0.009 arcsec, 3- $\sigma$ on time scales upwards of 15 minutes. As the pixels span 3.98 arcsec on each side, this translates to pointing precision of 0.75 millipixels, $1-\sigma$. This is $\sim 1000 \times$ better than that of the Hubble Space Telescope on a pixel scale. The compression scheme and the onboard storage allow Kepler to observe continuously for month-long intervals with brief interruptions to allow for downlinking the data to the ground.

The following sections describe some of the key challenges related to collecting and processing the science data and our efforts to mitigate them. Section 3 addresses the key challenge of monitoring the target stars as continuously as possible by minimizing both the number of planned interruptions to the observations as well as the time it takes to downlink the data from the spacecraft. Section 4 describes key challenges posed by nature in the form of stellar variability and by the subtle, but very important, thermal sensitivity of the instrument.

\section{MANAGING KEPLER'S DATA VOLUME}

One of the key challenges for Kepler is the management of the data volume. A full frame image (FFI) for Kepler has $101,744,160$ pixels, including 7,126,560 collateral pixels used for calibration read out from 84 CCD module outputs (2 per CCD). The individual 6.54-sec frames are co-added for 29.4 minutes (a Long Cadence interval). Consequently, the full, uncompressed image data acquired every day are $>300$ GB. The full frame images contain significantly more data than we can afford to store onboard the Solid State Recorder (SSR), which must be able to hold at least two months of data for robustness against a missed monthly downlink opportunity. The strategy for reducing the data volume consists of first retaining only the pixels of interest (POI) and then applying a lossless compression scheme to squeeze out the

\footnotetext{
${ }^{\ddagger}$ It appears to be impractical to detect Earth-size planets in the HZ of Sun-like stars by any means from the ground, given the small mass and size of such objects, which is why there are space missions proposed to detect them by transit, by ultra-high contrast imaging and by gravitational microlensing.
} 


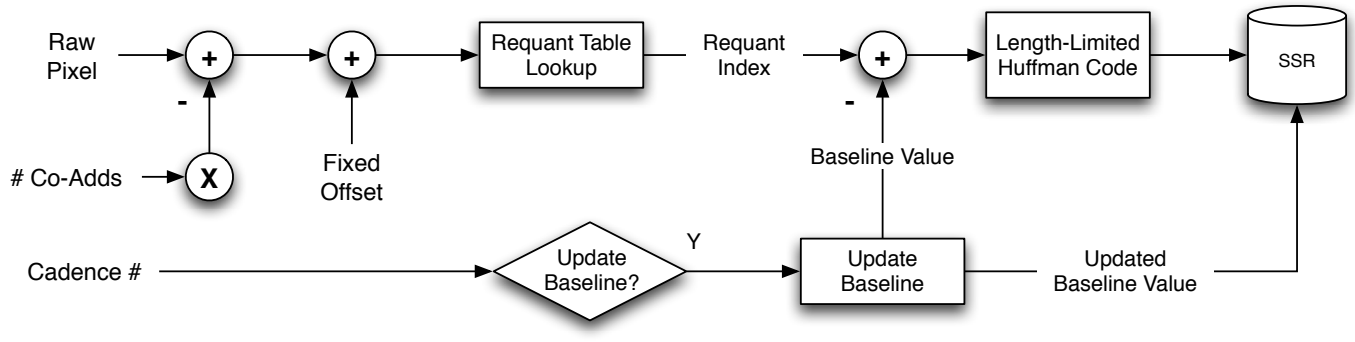

Figure 1. The flow of the compression algorithm implemented in flight software. The co-added pixel values are first shifted to a common zero point through the use of a fixed offset and the mean black level (bias). The shifted pixels are then mapped to the nearest neighbor in the requantization table that has larger steps between large pixel values and smaller steps between small pixel values so that the quantization noise is a fixed fraction of the intrinsic measurement uncertainty. A daily baseline value is subtracted and the residuals are entropically encoded using a length-limited Huffman code in order to squeeze out temporal redundancies in each pixel time series. The compressed bit stream is written to the Solid State Recorder for later downlink to the Deep Space Network.

temporal redundancies in the data stream. The responsibility for managing the data volume is distributed between the Science Operations Center (SOC) at NASA Ames Research Center, and Kepler's flight software. The SOC provides the compression tables and the target definitions, and the flight software uses this information to select the pixel data that are compressed and stored on the SSR. The Data Management Center at STScI decompresses the data on the ground prior to sending them to the SOC. The success of this approach depends on a number of factors.

\subsection{Discarding Pixels of Little or No Interest}

The first step in reducing the data volume is accomplished through the use of target definitions, which allow us to specify up to 170,000 Long Cadence (LC) stellar targets for 29.4-minute integrations which can occupy up to 5,440,000 pixels. Each definition contains a reference row and column along as well as an index into an aperture mask table onboard Kepler containing 1024 different masks that vary in shape and size. ${ }^{15} 512$ Short Cadence (SC) targets with up to 43,520 pixels can also be specified for 58.7-sec integrations ${ }^{\S}$. The KIC, together with models for the pointing behavior of the spacecraft and the point spread function (PSF), ${ }^{16}$ allows us to identify the pixels required for each stellar target based on an SNR analysis of the average scene surrounding each target star. ${ }^{15}$ More pixels are assigned to bright stars than to dim stars: LC targets are allocated an average of 32 pixels per star, while SC targets are allocated an average of 85 pixels per star. The aperture masks assigned to the targets don't contain much margin for error: a one-pixel halo is added to the pixels identified as essential to calibrating and making the photometric measurements. The masks are efficient: only $4 \%$ of the pixels addressed by the masks are not pixels of interest. ${ }^{15}$ The POI include sufficient pixels to monitor the background flux levels (up to 4500 per CCD output) and to allow us to correct for on-chip artifacts such as variations in the bias voltage added to the CCD output prior to digitization, and to monitor and remove the effects of the shutterless readout smear which occurs because Kepler operates without a mechanical shutter. ${ }^{17,18}$ No background pixels are collected at SC, and the smear and black (bias) collateral data are only collected at the SC intervals for the rows and columns containing SC target pixels.

The success of this approach to reduce the amount of data stored and transmitted by Kepler relies on several factors. We need to understand in great detail the scene around each potential target star so that we can render accurate synthetic images for the SNR calculation supporting the identification of the required pixels. This is supplied by the KIC, which contains $4.5 \times 10^{6}$ stars and their characteristics ${ }^{\pi}$. Generating the photometric scene for each target star requires knowledge of the PSF, and knowledge of exactly where the star is going to be throughout the three months of upcoming observations. The Pixel Response Function (PRF), essentially a 2-D representation of the interaction of the PSF with the sub-pixel sensitivity variations in the CCDs, allows the stellar images to be rendered accurately. How the celestial coordinates of the stars map to silicon coordinates is defined by the Focal Plane Geometry (FPG) model. These models are coupled to a heuristic, predictive model of the pointing behavior of the spacecraft in order to identify the POI. Obtaining the PRF and FPG models

\footnotetext{
${ }^{\S}$ Each Long Cadence interval consists of 30 Short Cadence intervals, and each Short Cadence consists of 9 co-added 6.54-sec integrations.

"Generating the KIC was a significant challenge in itself and required several observing seasons and a significant amount of effort to reduce the data and characterize the stars.
} 
was a major feat and resulted from a set of unique data collected and analyzed during the commissioning phase. ${ }^{16,19,20} \mathrm{~A}$ total of 121 15-minute LC frames were collected over a grid of 2-D offsets over \pm 0.5 pixels. These observations were made for 52,496 stars and used over-sized aperture masks with two halos to allow for uncertainties in the pre-launch FPG models. Approximately 200 of the brightest, unsaturated stars on each CCD readout channel were used to simultaneously solve for the super-resolution PRFs, which were allowed to vary in shape over each CCD readout channel, and for the FPG coefficients. The pointing model which plays an essential supporting role in these calculations assumes that once the ADCS locks onto the guide star, it acts to minimize the movement of the guide stars from their starting positions. This simple, heuristic pointing model has proven to be quite successful despite the fact that the detailed motion of the stellar images cannot be modeled by a static, rigid-body coordinate transform, due to small, thermally induced, non-uniform focus changes. The Target and Aperture Definition (TAD) pipeline component uses these models to analyze the average SNR of each target's flux measurement over the upcoming three months of observation. Note that differential velocity aberration causes the stars at the edges of the large $\sim 13.25^{\circ} \times 13.25^{\circ}$ field of view to move by up to $\sim 0.6$ pixels over 90 days so the aperture masks must be large enough to capture this motion.

Of equal importance to this task is the pipeline component, Photometer Data Quality (PDQ), which reconstructs the attitude of the spacecraft after each return to science data collection to confirm that each and every target star is within \pm 0.1 pixels or 0.398 arcsec of its expected location. ${ }^{21}$ If we can't achieve this accuracy then the target definitions will become invalid during the observations and we lose essential data. This is a daunting task: the focal plane is approximately 12,000 pixels across. The required precision is therefore 1 part in 120,000. This is comparable to the photometric precision required to detect Earth-size transits. If any target star falls outside this very small bull's-eye, then PDQ furnishes a pointing "tweak" in the form of a delta quaternion that represents the error between the desired pointing and the actual pointing reconstructed from the data. Upon request by the Kepler Science Office, this delta quaternion can then be uplinked and injected into the ADCS as an artificial pointing error that will be nulled out and therefore, nudge the spacecraft to the correct attitude. PDQ itself has far fewer pixels to work with than is available for normal science, as its data is provided through the low gain antennas at X-band. At the start of the mission, PDQ relied on no more than 96,000 pixels with about 300 star images from the focal plane array, and now only has access to $\sim 160$ stars. The number of stellar targets that PDQ receives for its attitude reconstruction will continue to drop as it drifts away from Earth and the strength of the radio link to the spacecraft diminishes. So far, PDQ has proven itself up to the task.

The use of the target definitions reduces the fraction of pixels that are stored on the SSR to $\sim 5 \%$ of that in a FFI. The raw data are represented as 23 bit words and the compressed data must occupy no more than 5.75 bits per pixel on average in order for the SSR to achieve adequate storage capacity. The uncompressed POIs are still a factor of 4 too large to store on the SSR. We can take advantage of two aspects of the digitized data to achieve the necessary level of compression: 1) the digitized pixel data for bright pixels are highly over-represented, and 2) the temporal redundancy in the observations. The former is handled by the requantization scheme described in Section 3.2, and the latter is addressed by a length-limited Huffman code discussed in Section 3.3.

\subsection{Requantization}

First, the 14-bit Analog-Digital-Converters (ADC) quantize the pixel measurements uniformly to $\approx 100 \mathrm{e}^{-} / \mathrm{ADU}^{\mathrm{N}}$, regardless of the intrinsic measurement noise of each observation. As a consequence, the bright pixels are over-represented due to shot noise, which varies as the square root of the number of photons as an $r m s$ value. For example, the well depth of the CCDs is approximately $1 \times 10^{6} \mathrm{e}^{-}$. Thus, after 270 exposures (an LC interval), the brightest pixels have $270 \times 10^{6} \mathrm{e}^{-}$ with a corresponding shot noise of $1,6432 \mathrm{e}^{-}$, and the quantization is at the $100 \mathrm{e}^{-}$level, ${ }^{22}$ so these pixels have a resolution that is 164 times smaller than the intrinsic measurement uncertainty. To reduce the degree to which bright pixels are over-represented, Kepler applies a software "compander" to redistribute the bits associated with the pixel measurements so as to make the ratio of the quantization noise to the total intrinsic noise a fixed value chosen to be $r=1 / 4$, called the requantization ratio. This is accomplished with a "requantization" table that is a list of allowed pixel values whose spacing increases as a function of the measured pixel value. The requantization algorithm replaces each pixel measurement with the index of the nearest pixel value in the requantization table (containing exactly $2^{16}$ entries) by the flight software, i.e., it is a simple look-up table with nearest neighbor interpolation.

What effect does requantization have on the total noise? We have

$$
\sigma_{\text {total }}^{2}=\sigma_{\text {intrinsic }}^{2}+\Delta_{Q}^{2} / 12=\sigma_{\text {intrinsic }}^{2}+r^{2} \sigma_{\text {intrinsic }}^{2}=\left(1+r^{2}\right) \sigma_{\text {intrinsic }}^{2}
$$



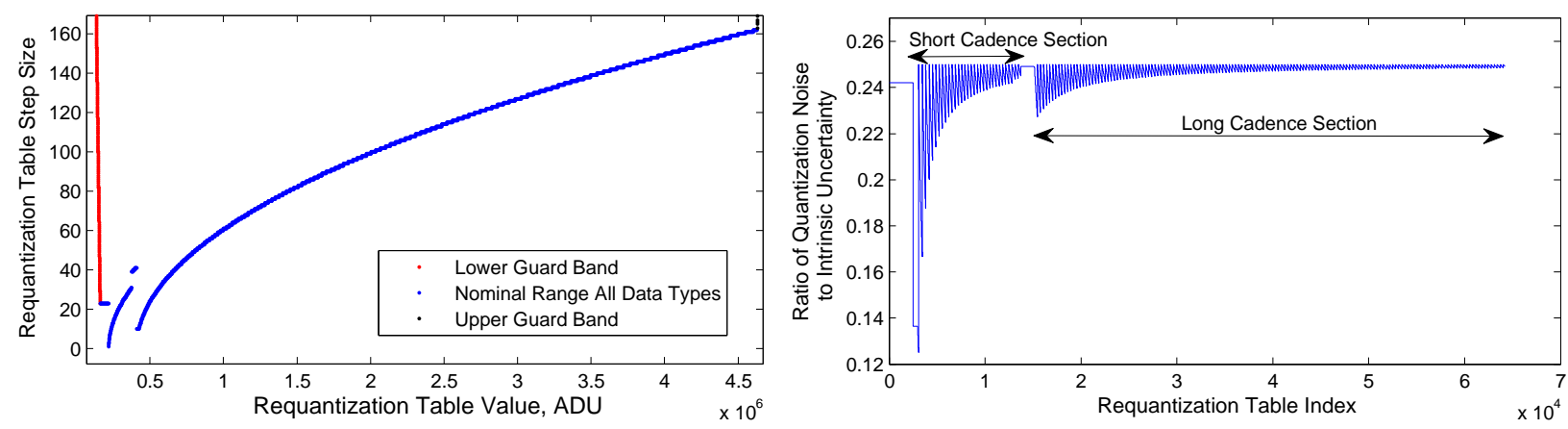

Figure 2. Left: The requantization table step sizes as a function of table index. The two main photometric data sections are shaped approximately like the square root function, with low middle and high guard band sections above, below and in between the short cadence and long cadence sections of the table. Right: A verification figure showing that all data types are quantized to no more than $1 / 4$ of their intrinsic uncertainties.

where $\sigma_{\text {total }}^{2}$ is the combination of the measurement uncertainty, $\sigma_{\text {intrinsic }}^{2}$, and the quantization noise, $\Delta_{Q}^{2} / 12$, due to a quantization step size of $\Delta_{Q} \cdot{ }^{23}$ The requantization increases the $r m s$ noise on the observations by $\sigma_{\text {total }} / \sigma_{\text {intrinsic }}-1=$ $\sqrt{1+r^{2}}-1=0.03$ for the choice $r=1 / 4$ a rather modest increase of 3\%. This is acceptable as this approach treats the quantization noise as a term in the noise budget and the requantization scheme allows us to trade a slight increase in the noise level of the photometric measurements for a large reduction in the data volume taken up by the measurements and the time required to downlink the data.

How do we build the requantization table? The concept is simple: start with the lowest photoelectron count expected in terms of digital counts, $p_{\min }$, and set the first value in the requantization table, $p_{1}$ to this value. Set the counter $i=1$. The intrinsic uncertainty in a pixel value, $p_{i}$, in ADU is given by

$$
\sigma_{p_{i}}^{2}=\left(p_{i}-n_{\text {co-adds }} \cdot \mu_{\text {black }}\right) / G_{j}+n_{\text {co-adds }} \cdot \sigma_{\text {read }}^{2},
$$

where $n_{\text {co-adds }}$ is the number of individual exposures that were summed to obtain the pixel value, including any spatial co-adds, $\mu_{\text {black }}$ is the mean black or bias level in ADU, $G_{j}$ is the gain (ADU/e ${ }^{-}$) for the CCD channel $j$, and $\sigma_{\text {read }}$ is the rms read noise in ADU (typically 1 ADU). Compute the step size for element, $i$, as

$$
\Delta_{Q}\left(p_{i}\right)=\max \left\{1,\left\lfloor\sqrt{12} r \sigma_{p_{i}}\right\rfloor\right\}
$$

where $\lfloor\cdot\rfloor$ is the floor operator. Then $p_{i+1}=p_{i}+\Delta_{Q}\left(p_{i}\right)$.

In practice, generating the requantization table for the flight segment is complicated by the fact that the bias voltages added to the CCD readouts vary significantly across the focal plane, and they vary spatially even within each CCD readout channel. ${ }^{22}$ The SOC furnishes a mean black table to the flight segment for this use in addition to the requantization table. The electronic gains also vary significantly over the 84 CCD readout channels. ${ }^{22}$ Moreover, the collateral data used to calibrate the photometric data for on-chip artifacts, such as the bias voltage (or black level) and the shutterless readout smear, are co-added spatially before being compressed. Also, there are two cadences for photometric data, Short Cadence, consisting of 9 summed readouts, and Long Cadence, consisting of 270 summed CCD readouts. The readout noise associated with SC and LC are quite different for pixels having the same value. Because of this, it is advantageous to design separate requantization table sections for the SC and LC data to help maximize the compressibility of the resulting data. The SOC generates a table combining the LC and SC sections, and uses a LC fixed offset of 419,400 ADU, and a SC fixed offset of 219,400 ADU that allow the flight software to tailor the requantization to the cadence type for each pixel measurement. Generating the requantization table requires a bit of bookkeeping.

Equations 2 and 3 are exercised for each table section to calculate the step size required by each SC or LC data type across the focal plane. The step size taken is the smallest one required, after which the process repeats until $p_{i}$ exceeds the expected range of the photometric measurements. This design allows for guard bands above, below, and between the two main requantization tables to ensure that drifts in the DC bias voltages don't result in variations in pixel values that fail to be captured by the requantized data because the input values fall outside the expected input range. Requantization reduces the size of the data from 23 bits to 16 bits per pixel per cadence, a substantial, but insufficient reduction in the data volume. 

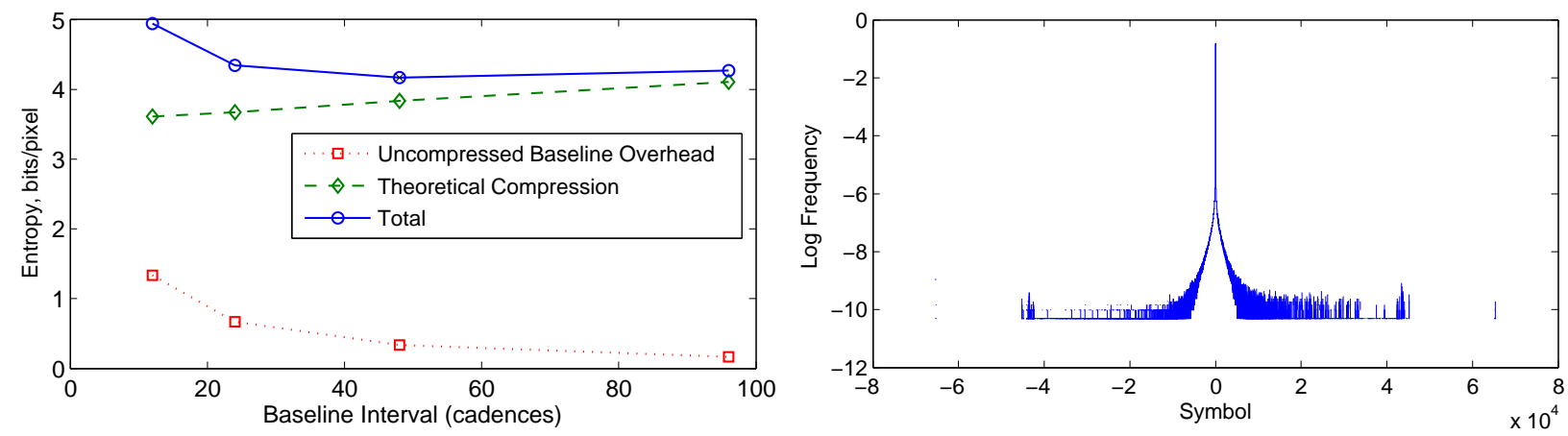

Figure 3. Left: The compression rate as a function of the baseline interval for the predictor for four different baseline intervals. The solid blue curve with circles is the total compression rate, while the dashed green curve with diamonds is the entropy for the compressed data and the dotted red curve with squares is the overhead cost associated with storing the baselines, which are not compressed. There is a shallow minimum at 48 cadences $(\sim 1$ day), which is the value selected for the baseline interval. Right: Histogram of the symbol frequencies corresponding to a baseline interval of 48 Long Cadences. The symbols can span the range from $-2^{16}+1$ to $2^{16}-1$. The compactness of the residuals dictates the compression that can be achieved. Over $99 \%$ of the pixel residuals are expected to be within 13 requantization levels of the daily baseline value.

\subsection{Length-Limited Huffman Coding}

The second part of the compression scheme takes advantage of the slowly varying nature of the scene imaged by Kepler to reduce the variance in each POI's time series by removing a predicted value for each POI. This prediction is furnished by a requantized measurement for each pixel made during a baseline cadence interval, and this baseline measurement is updated every 48 cadences (which is a software parameter) starting with the first cadence collected after return to science mode. The residuals from the baseline value are entropically encoded using a length-limited Huffman code ${ }^{24-26}$ that limits the code words to no more than 24 bits. Huffman codes compress data streams by representing common symbols with

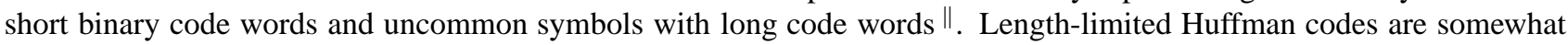
less efficient than non-length-limited codes, but the performance in the context of Kepler is more than adequate. The uncompressed baseline pixel values are stored onboard the SSR as well as the differences between the previous baseline value and the new baseline value for each pixel, which is compressed. This allows us to reconstruct the requantized pixel values from the bit stream both forward from the previous baseline as well as backwards from the following baseline in case a baseline measurement is lost or corrupted.

Generating a Huffman coding table requires that the frequencies of the symbols to be coded be quantified. In Kepler's case, we needed to generate and fly a compression table prior to flight for test purposes. To do so, we generated high fidelity, synthetic science data using the Kepler End-To-End Model (ETEM) ${ }^{27,28}$ and ran it through the Huffman Generator (HGN) pipeline where the the symbol histograms are generated for each of the $84 \mathrm{CCD}$ readout channels ${ }^{* *}$ for the list of baseline intervals specified on input. The Huffman Accumulator (HAC) pipeline then aggregated the results across the 84 module outputs and produced diagnostic plots and information so that we could determine whether the default baseline cadence interval produced adequate compression rates. Figure 3 shows that the default baseline interval of 48 Long Cadences is located approximately at the bottom of a very shallow minimum as the overhead associated with storing the uncompressed baseline interval data is traded against better compression for shorter baseline intervals. The predicted compression rate is 4.17 bits per pixel, which is significantly better than the requirement.

The original plan was to update the Huffman table once we were in orbit and had the opportunity to collect a sufficient amount of flight data. However, as the data in Figure 4 shows, this was not necessary. The Calibration (CAL) pipeline examines the original LC pixels that flow through it to be calibrated and calculates the achieved and theoretical entropies for tracking and trending purposes. ${ }^{29}$ As can be seen for the third quarter of observations (Q3), the difference between the achieved and theoretical entropies is only 0.13 bits per symbol, or $3 \%$, so in spite of the fact that ETEM does not simulate

\footnotetext{
${ }^{\|}$Huffman codes are ubiquitous and are used in various digital coding standards including mp3 and jpeg applications as well as in fax machines.

** Each CCD has two readout channels, or module outputs.
} 

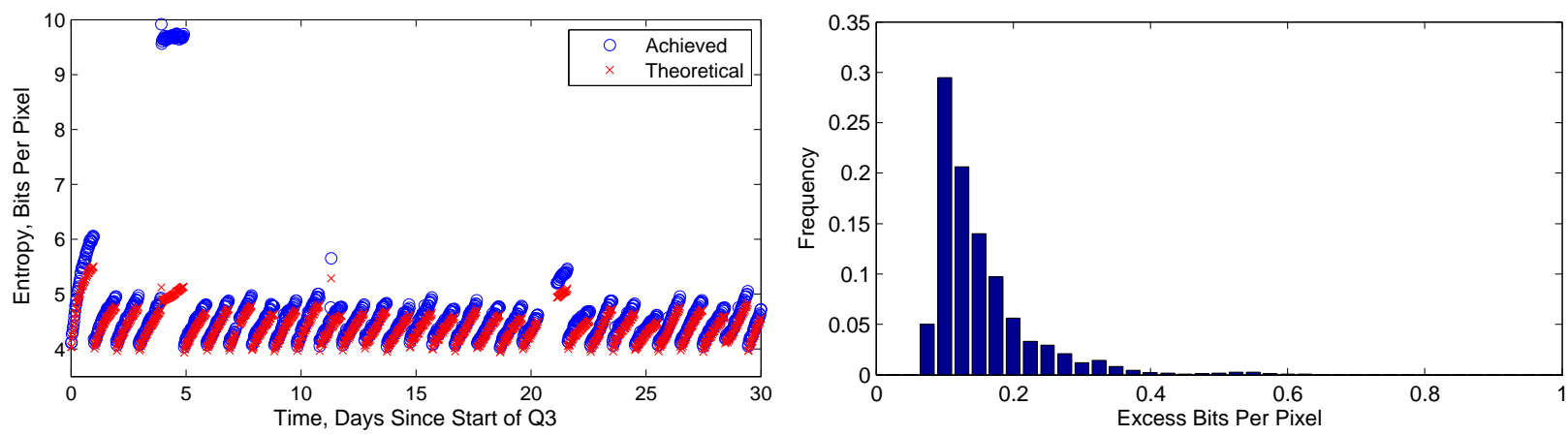

Figure 4. Left: Achieved and theoretical compression rates for the Q3 data set for the first 30 days of the data collection period. This data set spanned $\sim 90$ days and was the first "typical" quarter of observations as it was 3 months long and did not suffer from excess pointing drift, which necessitated pointing "tweaks" during Q2, or from excessive safe mode events. The effect of the predictor chosen for Kepler can be seen in the ramping up of the entropies over the course of each 48 -cadence interval $(\approx 1$ day) as the baseline values for each pixel go stale. There are some occasions when the baseline is anomalous, most likely due to an Argabrightening event when a piece of debris crosses the aperture and reflects light into the barrel of the telescope ${ }^{30}$ Right: Histogram of the differences between the achieved and the theoretical compression rates given the distribution of requantized pixel residuals. The median achieved compression rate is 4.4 bits per pixel, only 0.13 bits per pixel above the theoretical minimum for a perfectly tuned compression table. The achieved compression is within $3 \%$ of the theoretical minimum for the chosen compression algorithm.

highly variable, non-solar-like stars, of which there are several thousand to be found in the Kepler target catalog, we are achieving a level of compression, $\sim 4.4$ bits per pixel per cadence, that is very close to the optimum for the compression algorithm selected and implemented in flight and ground software.

\section{CHALlENGES TO PHOTOMETRIC PRECISION}

Since launch, Kepler has provided photometric time series of unprecedented quality and completeness which have in many respects surpassed the expectations of the scientists and engineers who designed and built the spacecraft. There have been some important facts that came to light after Kepler returned a significant amount of data. First, while the photometry has been unparalleled in quality, there appears to be excess photometric noise so that the mode of the precision for $K p=12$ G2V stars (solar analogs) on timescales of 6.5 hours is $30 \mathrm{ppm}$ rather than the desired goal of $20 \mathrm{ppm}$. The Kepler team has been studying the data to identify likely causes and has determined that the bulk of the increase in the observed photometric noise is due to stellar variability, ${ }^{31}$ which is about twice as high as expected based on $\mathrm{SOHO}$ observations of the Sun. ${ }^{3}$ This is an important discovery, both from the standpoint of understanding our Sun in the context of stars in general, and from that of designing future space transit photometry missions.

The fact that solar-like main-sequence stars exhibit more variability than the Sun on timescales relevant to transit detection does not prevent the Kepler Mission from achieving its goals, as increasing the duration of the mission to a period of 8 years will recover the originally scoped sensitivity. However, it does make it that much more important to deal with other challenges facing the attainment of $20 \mathrm{ppm}$ photometric precision, such as various systematic errors and instrumental signatures, which are not always adequately removed from Kepler light curves at present.

\subsection{Instrumental Signatures and Systematic Errors}

Although Kepler is in a very benign, Earth-trailing orbit, there are changes in the thermal state of the telescope as the Sun moves in relation to the telescope. There are also heaters on various spacecraft components used to condition the temperatures of these items within their required bounds. The telescope changes shape in response to the changing distribution of temperatures in the structure, and consequently, the focus of the instrument changes in subtle, but important ways. ${ }^{17,32}$ In fact, during the first quarter of science observation we noticed that a heater in the reaction wheel assembly for wheels 3 and 4 was cycling on and off in response to the fact that the box was becoming more shaded during the quarter and the changing temperature of the box was inducing perfectly correlated signatures in the science data at the pixel level. The shape of the telescope was deforming and changing the distance between the primary mirror and the focal plane assembly by $\sim 1 \mu \mathrm{m}$ in response to this heater. This is a very small change in the state of the telescope, but when the goal is to reach 

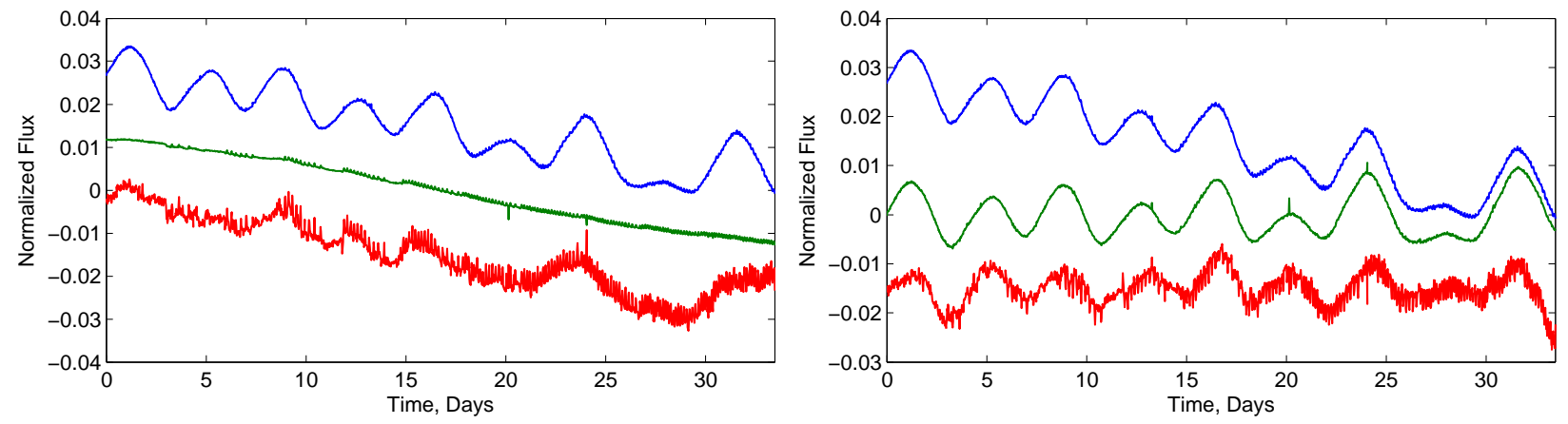

Figure 5. Light curves for one target on the most thermally sensitive CCD channel 2.1 in the Kepler focal plane for the Q1 observations. Left: The top curve (blue) is the simple aperture photometry flux time series, the middle (green) curve is the result of a MAP fit with Gaussian priors, and the bottom (red) curve is the result of a robust fit to the same basis vectors. Right: The top (blue) curve is the original flux, the middle (green) curve is the MAP-corrected flux, and the bottom (red) curve is the robust least-squares-corrected flux time series.

a photometric precision of $20 \mathrm{ppm}$, it is a significant change. The temperature limits of the thermostat were tightened up so that the cycle time was shortened below one half hour and the temperature swing was significantly reduced.

In the SOC Pipeline, the Pre-search Data Conditioning (PDC) module's task is to remove systematic errors from the raw flux time series. ${ }^{33}$ These include telescopic and spacecraft effects such as pointing errors, focus changes, and changing instrument properties. PDC co-trends each flux time series against ancillary engineering data, such as temperatures of the focal plane and electronics, pointing history, and focus variations, to remove signatures correlated with systematic errors. In general, PDC is doing a good job of correcting systematics for quiet, main-sequence stars, but it is not performing a uniformly good job for all targets, especially for those exhibiting significant intrinsic stellar variability. In some cases PDC removes or grossly distorts intrinsic variations and/or introduces short timescale variations that effectively add noise to the light curves, making it more difficult to detect signatures of transiting planets or to study astrophysics. We also see that in many cases the output light curves from PDC exhibit significant cross correlations, indicating that PDC is not removing all the instrumental signatures and systematics exhibited by the light curves. The basic problem is that PDC is fitting an incomplete model to the data and a maximum likelihood (ML) approach is happy to trade a reduction in bulk rms at the cost of introducing excess power on shorter time scales: ML is able to find a happenstance linear combination of basis vectors that match the gross profile of intrinsic stellar variations.

The solution we are implementing is a Bayesian Maximum A Posteriori (MAP) approach whereby we learn about the influence of the systematic errors by examining the behavior of the ensemble of light curves on each CCD readout channel. ${ }^{34}$ Figure 5 shows the results of applying a simple version of MAP to Q1 data when the heater on the reaction wheel assembly was active. We performed a singular value decomposition of the most-correlated light curves on this channel to obtain 10 basis vectors that were then robustly fitted to each stellar light curve. We formulated analytical priors from the distributions of the fit coefficients for each term and then applied a MAP fit. The MAP fit was able to "put the brakes" on the least-squares model fit so that highly variable stars were not over-fitted in an attempt to reduce the bulk rms, and intrinsic stellar variability was preserved to a high degree while instrumental systematics, such as a long term change in focus that reduced the brightness of the stars, and short term variations in focus caused by the reaction wheel heater, were effectively removed from the light curves. An empirical version of MAP is being implemented in the SOC PDC pipeline and will be completed and running on Kepler data sets by the end of 2011. We expect PDC-MAP to deliver light curves of much higher quality for asteroseismology and astrophysics investigations and for the exoplanet search.

\section{KEY SCIENCE RESULTS}

The Kepler Mission has been generating exciting science results right out of the box. The 10 days of continuous observations at the end of commissioning, now dubbed Q0, or Quarter 0, made it clear that detecting transits of giant planets was accomplished easily by eye. Examination of the light curve for HAT-P-7b revealed an $\approx 100 \mathrm{ppm}$ drop in brightness $180^{\circ}$ out of phase (an occultation) with the $0.6 \%$ transits that had been detected from the ground in 2008 before Kepler was launched. ${ }^{35}$ These small dips are the signature of the atmosphere of the planet heated to $\approx 2600 \mathrm{~K}$ by its proximity to its 
star, and were accompanied by phase variations in the light curve indicating that the transport of heat from the dayside to the nightside of the planet is inefficient. ${ }^{36}$ This light curve demonstrated unambiguously that Kepler can detect transiting planets and furthermore, can detect the weak signatures of Earth-like planets transiting sun-like stars.

The identification of hundreds of potential transiting planets in the initial Kepler observations followed quickly on the heels of the occultation of HAT-P-7b. By January of 2010, five planets were announced and hundreds of additional candidates awaited the following observing season for follow up observations and confirmation. The first system of multiple transiting planets was announced in August 2010, ${ }^{37}$ consisting of two Saturn-size planets conducting a coupled orbital dance near resonance with 19.24 and 38.91 day periods. These bodies exhibit mutual gravitational interactions in the timing of the transits observed by Kepler. These transit timing variations (TTVs) can be used to infer or constrain the masses of the transiting bodies, and can also indicate the presence of non-transiting planets that are perturbing the orbits of the transiting planets. The first unquestionably rocky planet was announced by the Kepler team in January of $2011 .{ }^{38}$ Radial velocity observations of this planet indicated it has a mass of $4.5 \mathrm{M}_{\oplus}$, so with a radius of $1.4 \mathrm{R}_{\oplus}$ this planet has a density of $8.8 \mathrm{~g} \mathrm{~cm}^{-3}$. Asteroseismology provided a much improved radius estimate for Kepler-10b's star. Kepler-11, an extremely compact system of 6 transiting planets, was discovered with 5 of the 6 planets exhibiting TTVs so that their masses could be estimated directly from the Kepler data. ${ }^{39}$ The planetary orbits in this system are highly coplanar.

Beyond the individual planet discoveries, the statistics of the Kepler results are accumulating to the point that we are learning about the nature and prevalence of planetary formation. Of the 1235 candidates identified by Kepler, ${ }^{1} 408$ of these planetary candidates are found in 170 multiple systems, demonstrating the flatness of planetary systems composed of small planets. Analysis of the single transiting candidates and the multiple transiting candidates suggests a relative absence of giant planets in close orbits for the multiple planet systems, and that the false positive rate for the multiple transit systems is significantly lower than that of the single transit planet candidates. ${ }^{40}$ The data release of February 2011 has led to a profusion of papers inferring the intrinsic planet frequency from the initial candidate planet catalog. While it is premature to extrapolate these initial findings to the ultimate prize, Earth-size planets orbiting in the habitable zone of solar-like stars, the initial results indicate that the frequency of planets varies inversely with their size, so that smaller planets are more common than giant planets down to at least $2 R_{\oplus} \cdot{ }^{1,41}$

As important as Kepler is for exoplanets, the asteroseismology community in turn is using this instrument as a nearideal platform to write new chapters in their field. Initial results from the Kepler data suggest that the long-held belief that asteroseismology can reveal the secret inner workings of stars is being confirmed today. ${ }^{42}$ The Kepler data allow us to quantify global properties of the observed stars, such as radius and age, through observations of normal modes of oscillation. One of the most exciting recent results is the ability of Kepler to distinguish between giant stars that are still burning hydrogen in a shell surrounding the accumulating helium core, and those giants that have initiated helium burning in the core. ${ }^{43}$ Finally, the Kepler data are also being used to study a wide variety of oscillating and pulsating stars, including RR Lyr stars, which can more than double their brightness every 12 hours, as well as cataclysmic variable stars.

\section{CONCLUSIONS}

The Kepler Mission is solving an astonishing array of astrophysical mysteries with its unprecedented photometric precision and near continuous observations. The success of Kepler is the result of many years of hard work and effort on the part of the thousands of individuals who contributed to the design, implementation, and operation of the instrument and spacecraft and of those who process and analyze the resulting data. Twenty-five years ago the Kepler concept was a sci-fi fantasy that only a few individuals, notably the Science Principal Investigator, William J. Borucki, believed in. The protagonists in this narrative persevered and overcame the many daunting obstacles in the path to the ultimate goal by meeting each challenge as it arose and mustering the resources and talent to solve each problem in turn.

By their nature, transit survey missions rely on collecting time series image data. Such missions must rely on the stability of their observing platforms to achieve the ultra high precision needed to discover small planets. The methodology used by Kepler to reduce the data volume to a manageable level, and then to compress the data without loss can be readily applied to future missions to reduce the storage required per sample and to minimize the transmission time required to downlink the original data. It is extremely important to minimize the perturbations to the instrument on timescales from 0.01 to several days. Even in Kepler's benign orbital environment, identifying and removing instrumental systematic errors is a real challenge for such sensitive instruments, and the innovative use of Bayesian methods should be explored. 
ESA's PLATO Mission, ${ }^{44}$ an ambitious transit survey to discover Earth-size planets around the closest and brightest main-sequence stars may be selected for flight by the end of 2011. It would launch in 2017 or 2018 into an orbit at L2 to survey several large $>800 \mathrm{deg}^{2}$ fields of view with an instrument composed of $\sim 30$ small telescopes. Also, at least two transit surveys have been proposed to NASA's current Explorer Mission competition. MIT has proposed the Transiting Exoplanet Survey Satellite (TESS) mission, ${ }^{45}$ an optical, all-sky transit survey satellite to be launched into orbit in 2016 for a two-year mission with an instrument composed of multiple telescopes with disjointed fields of view. JPL has also proposed an infrared transit survey mission called ELEKTRA to the Explorer Program. ${ }^{46}$ These future missions will face many of the same challenges and will surely benefit from the successes of and the lessons learned from the Kepler Mission.

\section{ACKNOWLEDGMENTS}

The authors thanks all the engineers, managers, technicians and support staff at Ball Aerospace Technologies Corporation for delivering such a fine instrument to the world. I thank the Kepler Science Team for bringing the nascent science in the data to life and the Science Operations Center team for developing the science processing pipeline. I thank scientific programmers Hema Chandrasekaran for coding the requantization table and Huffman code generation table, and Joe Twicken (SETI Institute) for coding the Huffman histogram generator and accumulator software. I thank Bill Wohler for writing the java code for these components. Funding for the Kepler Mission is provided by NASA's Science Mission Directorate.

\section{REFERENCES}

[1] Borucki, W. J.,et al., "Characteristics of planetary candidates observed by Kepler. II. Analysis of the first four months of data," ApJ 736(1), 19 (2011).

[2] Jenkins, J. M., D. A. Caldwell, and Borucki, W. J., "Some tests to establish confidence in planets discovered by transit photometry," ApJ 564, 495 (2000).

[3] Jenkins, J. M., "The impact of stellar variability on the detection of transiting terrestrial planets", ApJ 575, 493 (2002).

[4] Young, A. T., "Photometric error analysis. VI. Confirmation of Reiger's theory of scintillation," AJ 72, 747-753 (1967).

[5] Koch, D. G., et al., "Kepler Mission design, realized photometric performance, and early science," ApJL 713(2), L79-L86 (2010).

[6] Robinson, L. B., Wei, M. Z., Borucki, W. J., Dunham, E. W., Ford, C. H., and Granados, A. F., "Test of CCD Precision Limits for Differential Photometry," PASP 107, 1094 (1995).

[7] Jenkins, J. M., Borucki, W. J., Dunham, E. W. and McDonald, J. S., "High Precision Photometry with Back-Illuminated CCDs," ASP Conf. Ser., 119, 227-280 (1997).

[8] Koch, D. G., et al., "CCD photometry tests for a mission to detect Earth-size planets in the extended solar neighborhood," Proc. SPIE 4013, 508-519 (2000).

[9] Jenkins, J. M., Witteborn, F., Koch, D. G., Dunham, E., Borucki, W. J., Updike, T. F., Skinner, M. A., and Jordan, S. P., "Processing CCD images to detect transits of Earth-sized planets: Maximizing sensitivity while achieving reasonable downlink requirements," Proc. SPIE 4013, 520-531 (2000).

[10] Borucki, W., et al., "Kepler planet-detection mission: introduction and first results," Science 327, 977-980 (2010).

[11] Ebbets, D., Atcheson, P., Stewart, C., Spuhler, P., "Optical performance of the 100 sq deg FOV telescope for NASA's Kepler exoplanet mission," Proc. SPIE 8146, 8146-15, this volume (2011).

[12] Ebbets, D., Argabright, V., VanCleve, J., Caldwell, D. "In-flight performance of the $96 \mathrm{Mpx}$ focal plane for NASA's Kepler mission," Proc. SPIE 8146, 8146-16, this volume (2011).

[13] Batalha, N. M., et al., "Selection, prioritization, and characteristics of Kepler target stars," ApJL 713(2), L109-L114 (2010).

[14] Brown, T. M., Latham, D. W., Everett, M. E., and Esquerdo, G. A., "Kepler input catalog: Photometric calibration and stellar classification," AJ, in press, arXiv:1102.0342 (2011).

[15] Bryson, S. T., et al., "Selecting pixels for Kepler downlink," Proc. SPIE 7740, 77401D (2010).

[16] Bryson, S. T., et al., "The Kepler pixel response function,” ApJL 713(2), L97-L102 (2010).

[17] Jenkins, J. M., et al., “Overview of the Kepler science processing pipeline," ApJL 713(2), L87-L91 (2010).

[18] Quintana, E. M., et al., "Pixel-level calibration in the Kepler Science operations center pipeline," Proc. SPIE 7740, $77401 X(2010)$. 
[19] Tenenbaum, P., and Jenkins, J. M., "Focal plane geometry characterization of the Kepler Mission" Proc. SPIE 7740, $77401 \mathrm{C}(2010)$.

[20] Haas, M., et al., “Kepler science operations,” ApJL 713(2), L115-L119 (2010).

[21] Chandrasekaran, H., et al., "Semi-weekly monitoring of the performance and attitude of Kepler using a sparse set of targets," Proc. SPIE 7740, 77401B (2010).

[22] Van Cleve, J., and Caldwell, D. A., [Kepler Instrument Handbook, KSCI 19033-001], NASA Ames Research Center, Moffett Field, CA (2009).

[23] Papoulis, A., [Probability, Random Variables, and Stochastic Processes], McGraw Hill, New York (1984).

[24] Huffman, D. A., “"'A Method for the Construction of Minimum-Redundancy Codes," Proc. Inst. Radio. Eng. 40, 1098-1101 (1952).

[25] Larmore, L. L., and Hirschberg, D. S. , "A fast algorithm for optimal length-limited Huffman codes", J. Assoc. Comput. Mach. 37(3), 464-473 (1990).

[26] Katajainen, J., Moffat, A., and Turpin, A., "A fast and space-economical algorithm for length-limited coding”, Proc. of the 6th Int. Symp. on Algorithms and Computation, 1004, 12 - 21 (1995).

[27] Jenkins, J. M., Peters, D. J., and Murphy, D. W., "An efficient end-to-end model for the Kepler photometer," Proc. SPIE 5497, 202-212 (2004).

[28] Bryson, S. T., Jenkins, J. M., Peters, D. J., Tenenbaum, P. P., Klaus, T. C., Gunter, J. P., Cote, M. T., and Caldwell, D. A., "The Kepler end-to-end model: creating high-fidelity simulations to test Kepler ground processing," Proc. SPIE 7738, 773808 (2010).

[29] Li, J., et al., "Photometer performance assessment in Kepler science data processing," Proc. SPIE 7740, 77401T (2010).

[30] Witteborn, F. C., Van Cleve, J., Borucki, W. J., Argabright, V. S., and Hascall, P., “ Debris sightings in the Kepler field," Proc. SPIE 8151, 8151-42 (2011).

[31] Gilliland, R. L., et al., "Kepler Mission stellar and instrument noise properties," ApJ, in press, arXiv:1107.5207 (2011).

[32] Jenkins, J. M., et al., "Initial characteristics of Kepler long cadence data for detecting transiting planets," ApJL 713(2), L120-L125 (2010).

[33] Twicken, J. T., Chandrasekaran, H., Jenkins, J. M., Gunter, J. P., Girouard, F., Klaus, T. C., "Presearch data conditioning in the Kepler science operations center pipeline," Proc. SPIE 7740, 77401U (2010).

[34] Jenkins, J. M., Smith, J. C., Tenenbaum, P., Twicken, J. T., "Planet detection: the Kepler Mission," [Advances in Machine Learning and Data Mining for Astronomy], Chapman and Hall/CRC Press, in press (2011).

[35] Pál, A., et al., "HAT-P-7b: An extremely hot massive planet transiting a bright star in the Kepler field," ApJ 680(2), 1450-1456 (2008).

[36] Borucki, W. J., et al., “Kepler's optical phase curve of the exoplanet HAT-P-7b,” Science 325, 709 (2009).

[37] Holman, M. et al., "Kepler-9: A system of multiple planets transiting a sun-Like star, confirmed by timing variations,", Science 330, 51 (2010).

[38] Batalha, N. M., et al., “Kepler's first rocky planet: Kepler-10b," ApJ 729, 27 (2011).

[39] Lissauer, J. J., et al., "A closely packed system of low-mass, low-density planets transiting Kepler-11,” Nature 470, 53-58.

[40] Latham, D., et al., "A first comparison of Kepler planet candidates in single and multiple systems," ApJL 732, L24 (2011).

[41] Howard, A. W., et al., "Planet occurrence within 0.25 AU of solar-type stars from Kepler" ApJ, arXiv:1103.2541 (2011).

[42] Gilliland, R. L., et al., "Kepler asteroseismology program: Introduction and first results," PASP 122, 131-143 (2010).

[43] Bedding, T. R., et al., "Gravity modes as a way to distinguish between hydrogen- and helium-burning red giant stars," Nature 471, 608-611 (2011).

[44] Claudi, R., "A new opportunity from space: PLATO mission," Ap\&SS 328, 319-323 (2010).

[45] Ricker, G., et al., "Transiting Exoplanet Survey Satellite (TESS)," BAAS 42, 459 (2010).

[46] Beichman, C. and the ELEKTRA Team, "The ELEKTRA explorer mission to find transiting earth like planets," presented at the Strange New Worlds Conference held in Flagstaff AZ (2011).

xxiv 


\title{
Large Binocular Telescope Adaptive Optics System: New achievements and perspectives in adaptive optics
}

\author{
S. Esposito*a A. Riccardi ${ }^{a}$, E. Pinna ${ }^{\mathrm{a}}$, A. Puglisi ${ }^{\mathrm{a}}$, F. Quirós-Pacheco ${ }^{\mathrm{a}}$, C. Arcidiacono ${ }^{\mathrm{a}}$,

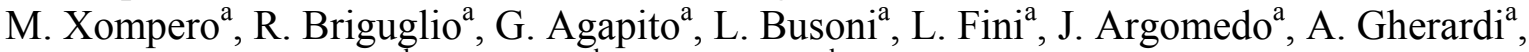 \\ G. Brusa ${ }^{b}$, D. Miller ${ }^{b}$, J. C. Guerra ${ }^{b}$, P. Stefanini ${ }^{a}$, P. Salinari ${ }^{a}$ \\ ${ }^{a}$ INAF - Osservatorio Astrofisico di Arcetri, Largo E. Fermi 5, 50125 Firenze, Italy; \\ ${ }^{b}$ LBT Observatory, Univ. of Arizona, 933 North Cherry Ave., Tucson AZ 85721, USA
}

\begin{abstract}
The Large Binocular Telescope (LBT) is a unique telescope featuring two co-mounted optical trains with $8.4 \mathrm{~m}$ primary mirrors. The telescope Adaptive Optics (AO) system uses two innovative key components, namely an adaptive secondary mirror with 672 actuators and a high-order pyramid wave-front sensor. During the on-sky commissioning such a system reached performances never achieved before on large ground-based optical telescopes. Images with 40mas resolution and Strehl Ratios higher than $80 \%$ have been acquired in $\mathrm{H}$ band $(1.6 \mu \mathrm{m})$. Such images showed a contrast as high as $10^{-4}$. Based on these results, we compare the performances offered by a Natural Guide Star (NGS) system upgraded with the state-of-the-art technology and those delivered by existing Laser Guide Star (LGS) systems. The comparison, in terms of sky coverage and performances, suggests rethinking the current role ascribed to NGS and LGS in the next generation of AO systems for the 8-10 meter class telescopes and Extremely Large Telescopes (ELTs).
\end{abstract}

Keywords: Large Binocular Telescope, high-order adaptive optics, pyramid sensor, adaptive secondary mirror.

\section{INTRODUCTION}

The purpose of Adaptive Optics (AO) is to correct wave-front aberrations of the electromagnetic waves received from a ground-based telescope after propagation through the atmosphere obtaining an image quality reached otherwise only with space-based telescopes. The Large Binocular Telescope ${ }^{[1]}$ (LBT) features two $8.4 \mathrm{~m}$ optical trains, each one of them fully integrated with AO correction provided by the two Adaptive Secondary Mirror (ASM) units with 672 actuators. The First-Light AO (FLAO) system ${ }^{[2]}$, designed for each optical train, is a Natural Guide Star (NGS) AO system featuring a modulated pyramid Wave-Front Sensor (WFS) with a maximum pupil sampling of $30 \times 30$ subapertures. A so fine spatial sampling is new for astronomical AO typically working in a photon starving regime. The spatial sampling is difficult to be realized also because of the technical limitations imposed by the limited degrees of freedom available on wave-front correctors. In the compromise between correction performance and available light, the efficiency of wavefront sensors plays a dominant role. In our system, the use of state-of-the-art devices such as the pyramid WFS and the adaptive secondary mirror offers the optimal playground for very high Strehl Ratio (SR) corrections on the bright end, and high efficiency use of reference starlight photons on the faint one. As we will show in this paper, we have obtained $>80 \%$ correction @ $1.6 \mu \mathrm{m}$ on a bright star (up to $M_{R} \sim 9$ ), and closed efficiently the loop on a $\sim 17^{\text {th }}$ magnitude star with $\sim 15$ photons per subaperture per frame ( $\sim 500$ in the whole pupil).

The on-sky commissioning of the FLAO\#1 system (installed on the right side of the telescope) started in May 2010 and will be completed in the fall of 2011. The installation of the FLAO\#2 system on the left side of the telescope started in August 2011. The two FLAO systems will provide AO correction on the bent-Gregorian focal stations serving the LUCIFER spectro-imagers ${ }^{[4]}$.

We will present in this paper the on-sky performance analysis of the FLAO\#1 system based on the data collected during the commissioning nights. After a brief system overview, we will present and discuss in the paper the performance achieved during the commissioning campaign. Finally we will present some considerations for future AO systems based on the results achieved at LBT.

*esposito@arcetri.astro.it; phone +39 0552752 309; fax +39 0552752292

Astronomical Adaptive Optics Systems and Applications IV, edited by Robert K. Tyson, Michael Hart, Proc. of SPIE Vol. 8149, 814902 - (C) 2011 SPIE · CCC code: 0277-786X/11/\$18 · doi: 10.1117/12.898641 


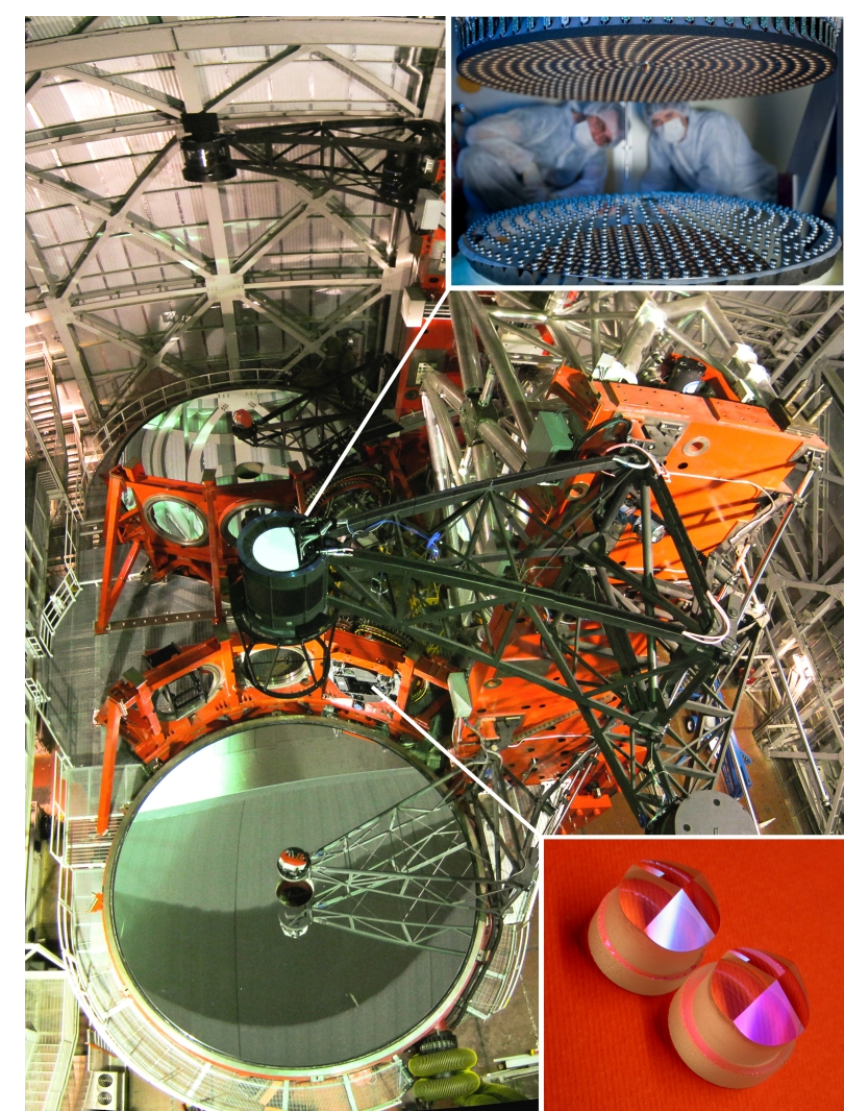

Figure 1. FLAO\#1 system installed on the right-side of the Large Binocular Telescope. The Acquisition, Guiding and Wavefront sensing (AGW) unit containing the pyramid WFS is located on the front-right bent-Gregorian focal station. In this photograph the ASM unit features the retro-reflector optics used for system calibrations mounted below it.

\section{FLAO SYSTEM OVERVIEW}

We will briefly review in this section the general characteristics of the FLAO\#1 system as it was commissioned to the telescope (Figure 1). A detailed description of the system optical design and laboratory characterization can be found in the cited papers ${ }^{[2][3]}$.

The ASM is equipped with a Zerodur thin shell of $1.6 \mathrm{~mm}$ thickness and $911 \mathrm{~mm}$ diameter. It relies on 672 contactless voice-coil force actuators to shape the thin shell with a maximum stroke of $\sim 100 \mu \mathrm{m}$. The settling time ranges from 0.7 to $1 \mathrm{~ms}$, and the best figuring error obtained at the telescope is $\sim 28 \mathrm{~nm}$ RMS in wavefront, using approximately the $25 \%$ of the forces range ${ }^{[5]}$. The FLAO\#1 commissioning results have been obtained with a total of 641 actuators. The contactless technology allows to software deactivate the 31 defective actuators without introducing hard points on the shell.

The pyramid $\mathrm{WFS}^{[6]}$ features a maximum of $30 \times 30$ subapertures to sample the mirror shape. The equivalent sampling on the primary mirror is $0.28 \mathrm{~m}$ which matches the Fried parameter $\left(r_{0}\right)$ obtained under good seeing conditions at the WFS central wavelength of $\lambda_{s}=750 \mathrm{~nm}$. The WFS camera uses a fast readout E2V CCD39 unit with 80x80 pixels and a maximum frame rate at full frame of $f_{s}=1 \mathrm{kHz}$.

The temporal and spatial sampling of the WFS can be adjusted as a function of $M_{R}$, the equivalent R-magnitude of the guide star (§3.1). In particular, the number of subapertures across the pupil diameter can be easily changed by choosing one of the on-chip CCD binning modes. In this way, the telescope pupil can be sampled with $30 \times 30,15 \times 15,10 \times 10$, or $\sim 7 \times 7$ subapertures when using, respectively, binning modes \#1, \#2, \#3, or \#4. The sensitivity of the WFS can also be adjusted by means of the pyramid (circular) modulation. 
Table 1. Typical system configurations used on sky as a function of the equivalent R-magnitude of the GS $\left(M_{R}\right)$. The system parameters are: the binning mode, the temporal sampling frequency $\left(f_{s}\right)$, the number of controlled modes $\left(n_{\text {mod }}\right)$, and the pyramid modulation. Median RON values measured for each configuration are also listed.

\begin{tabular}{|c|c|c|c|c|c|c|}
\hline $\boldsymbol{M}_{\boldsymbol{R}}$ & $\begin{array}{c}\text { Binning } \\
\text { mode }\end{array}$ & $\begin{array}{c}\text { Pupil sampling } \\
\text { (\# subaps.) }\end{array}$ & $\begin{array}{c}\boldsymbol{f}_{\boldsymbol{s}} \\
(\mathbf{H z})\end{array}$ & $\boldsymbol{n}_{\text {mod }}$ & $\begin{array}{c}\text { Pyr. mod. } \\
\left( \pm \lambda_{s} / \boldsymbol{D}\right)\end{array}$ & $\begin{array}{c}\text { RON } \\
\left(\boldsymbol{\sigma}_{\boldsymbol{e}} \mathbf{)}\right.\end{array}$ \\
\hline$M_{R} \leq 8.0$ & 1 & $30 \times 30$ & 990 & 500 & 2.0 & 10.5 \\
\hline$M_{R} \leq 10.0$ & 1 & $30 \times 30$ & 990 & 400 & 3.0 & 10.5 \\
\hline $10.0<M_{R}<13.5$ & 2 & $15 \times 15$ & $990 \leq f_{s} \leq 300$ & 153 & 3.0 & 6.4 \\
\hline $13.5 \leq M_{R}<14.5$ & 3 & $10 \times 10$ & $500 \leq f_{s} \leq 200$ & 66 & 6.0 & 4.5 \\
\hline $14.5 \leq M_{R}<16.5$ & 4 & $\sim 7 \times 7$ & $400 \leq f_{s} \leq 100$ & 36 & 6.0 & 4.6 \\
\hline $16.5 \leq M_{R}<18.0$ & 4 & $\sim 7 \times 7$ & 100 & 10 & 6.0 & 4.6 \\
\hline
\end{tabular}

The typical system parameters used on sky as a function of $M_{R}$ are summarized in Table 1. Also in this table are listed the actual Read-Out Noise (RON) median values measured for each system configuration.

The FLAO control system implementation is based on a modal approach. To be more effective in correcting the turbulence, the modal control basis uses Karhunen-Loève $(\mathrm{KL})$ modes fitted by the $\mathrm{ASM}^{[7]}$. The temporal controller is a modal integrator. Since the optimal integrator gains strongly depend on the atmospheric conditions, a procedure to automatically estimate the optimal gains has been developed. This procedure is run just after the Guide Star (GS) is acquired. Currently, gain values are not further changed during normal AO operation.

The modal interaction matrices between the ASM and the WFS (one for each pair of binning mode and pyramid modulation values) were calibrated at the telescope on daytime with the use of the retro-reflector optics ${ }^{[2]}$ installed below the ASM permitting the calibration beam to reach back the WFS unit (Figure 1).

\section{OBSERVING CONDITIONS}

The maximum performance that can be attained with an AO system is limited by several factors, the most important being the total received flux from the GS, the atmospheric turbulence conditions (i.e. the seeing value, and the wind speed), the separation between the object of interest and the GS, the read-out nosie of the WFS detector, and the telescope vibrations. We will focus in this paper on the on-axis performance results of the FLAO\#1 system in terms of the $\mathrm{SR}$ in $\mathrm{H}$ band as a function of the star magnitude and the atmospheric seeing value. We will describe in this section how we have estimated these two important parameters.

\subsection{Equivalent star magnitude in $R$ band}

The FLAO's pyramid WFS is sensitive to a broadband encompassing R and I bands. On the lower bound, the passband is limited by a dichroic sending all the light above $600 \mathrm{~nm}$ to the WFS. On the upper bound, the quantum efficiency curve of the WFS detector - a deep-depletion CCD39- limits the passband to $~ 950 \mathrm{~nm}$. Therefore, the luminosity of the GS in this band, 600-950nm, drives the AO correction capabilities.

For the purpose of comparing the performance of the FLAO system obtained with different reference stars, we will use the concept of an "equivalent" star magnitude in R band computed as:

$$
M_{R}=-2.5 \log _{10}\left[\frac{h \cdot c}{\tau_{s y s} \cdot \lambda_{s} \cdot e_{0}} n_{p h}\right]
$$

where $n_{p h}$ stands for the number of photons per squared-meter per second [ph $\left.\mathrm{m}^{-2} \mathrm{~s}^{-1}\right]$. This quantity can be easily estimated from the acquired WFS CCD39 frames. The constants in Eq. (1) are: $h$, the Planck constant; $c$, the light velocity; $e_{0}=1.76 \cdot 10^{-8} \mathrm{~W} \mathrm{~m}^{-2} \mu \mathrm{m}^{-1}$, the zero-magnitude brightness in the Johnson R band; $\lambda_{s}=750 \mathrm{~nm}$, the WFS central wavelength; and $\tau_{s y s}$, the overall optical transmission (Telescope + WFS board + CCD39) in the spectral band 600-950nm. 

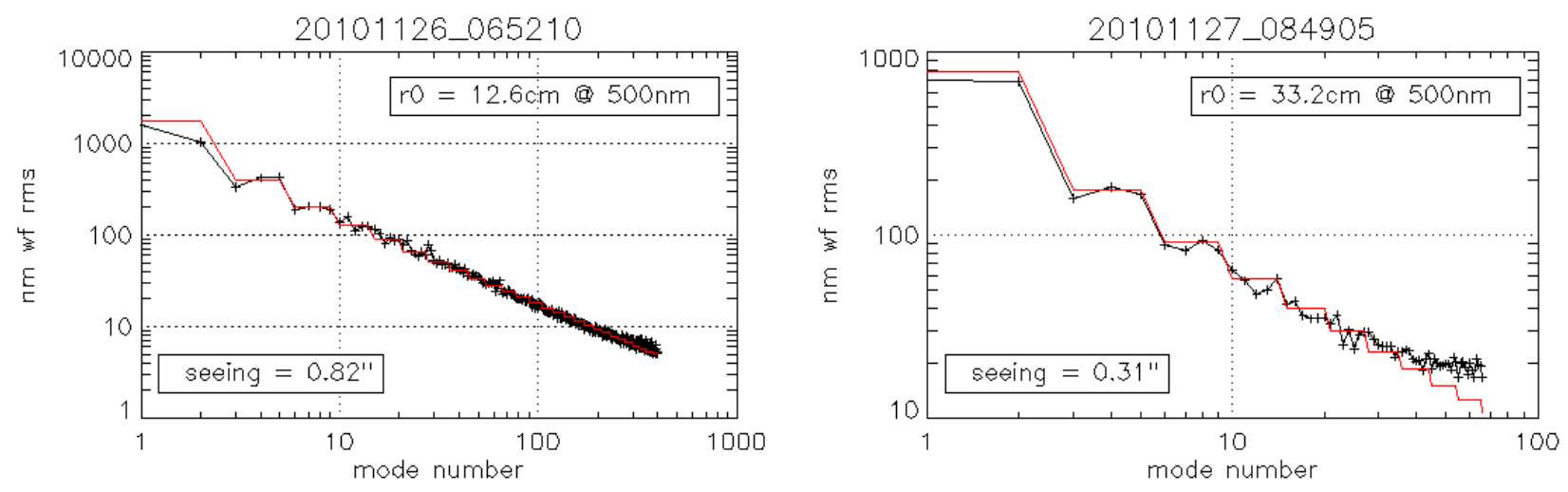

Figure 2. Example of $r_{0}$ fitting from reconstructed OL modes. (Crosses) Estimated OL modal variances; (Red line) Theoretical modal variance distribution. (Left) Case of bin\#1, 400 controlled modes. (Right) Case of bin\#3, 66 controlled modes.

\subsection{Estimation of the seeing value}

In order to have an estimate of the turbulence conditions during a given observation, LBT uses a Differential Image Motion Monitor (DIMM) installed on top of the telescope mount, and an anemometer installed on top of the telescope enclosure. An additional estimate of the seeing value can be found using the AO system telemetry data. This on-line seeing estimation method is based on the reconstruction of Open-Loop (OL) modes (i.e. the modal decomposition of the turbulence) from applied ASM commands and residual WFS signals acquired during the closed-loop operation of the AO system $^{[8]}$. Once the OL modes are available, the Fried parameter $\left(r_{0}\right)$ can be estimated from the OL modal variance distribution, from which the seeing value can be simply computed as $s(\lambda)=0.98 \lambda / r_{0}$ (by convention $s(\lambda)$ quoted at $\lambda=500 \mathrm{~nm})$.

Figure 2 shows the seeing estimation results for two particular cases. The plots show the time-variance distribution of the reconstructed OL modes, and the best-fit theoretical (Kolmogorov) variance distribution of Zernike modes. In order to avoid the uncertainty of the outer scale of the turbulence $\left(L_{0}\right)$ and the residual telescope vibrations, the first three radial orders are not considered in the fitting. Nor we have considered the last two radial orders, most likely affected by aliasing. Despite the good fitting achieved in both cases shown in Figure 2, we should note that the seeing estimation for the case of binning \#3 and 66 controlled modes is actually under-estimated. Indeed, the mismatch between the PSF size during the calibration of the interaction matrices (diffraction-limited) and the actual PSF size in closed-loop operation at WFS wavelengths is responsible for the underestimation of the modal residuals, which in general leads to an underestimation of the seeing value. We have found from our data analysis that this underestimation is non-negligible for datasets taken with star magnitudes $M_{R}>13$. We are currently working on an improvement of the on-line seeing estimation method in which the modal residuals are iteratively estimated.

\section{ON-SKY COMMISSIONING RESULTS}

We will now present the overall performance analysis of the FLAO\#1 system based on the data collected during the commissioning nights. The SR was estimated from the long-exposure PSFs measured with the InfraRed Test Camera ${ }^{[9]}$ (IRTC) using an H-band filter with an effective central wavelength of $1.60 \mu \mathrm{m}$. Total integration times varied from $\sim 30 \mathrm{~s}$ for high-flux PSF measurements up to $\sim 120$ s for the low-flux ones. The camera was operated in the narrow Field-ofView (FoV) mode with a pixel scale of 10 mas/pixel.

Figure 3 summarizes the performance results attained by the FLAO\#1 system. A total of 597 Strehl Ratio estimations at star magnitudes from $\sim 7.5$ to $\sim 18$ are shown in the plot. Seeing values estimated from AO real-time data are coded in color. Points $\left(M_{R}>13\right)$ for which seeing values are strongly under-estimated with this method are shown in black. Figure 3 also indicates which system configuration (i.e. binning mode) has been used for each acquisition. Finally, the expected performance estimated from numerical simulations and for different seeing values (0.6", 0.8 ", 1.0 ", 1.2 ", and 1.5") is also shown in the plot. It is important to note that the measured SR values are in accordance with the simulated ones, showing that the FLAO\#1 system meets the expected performance. 


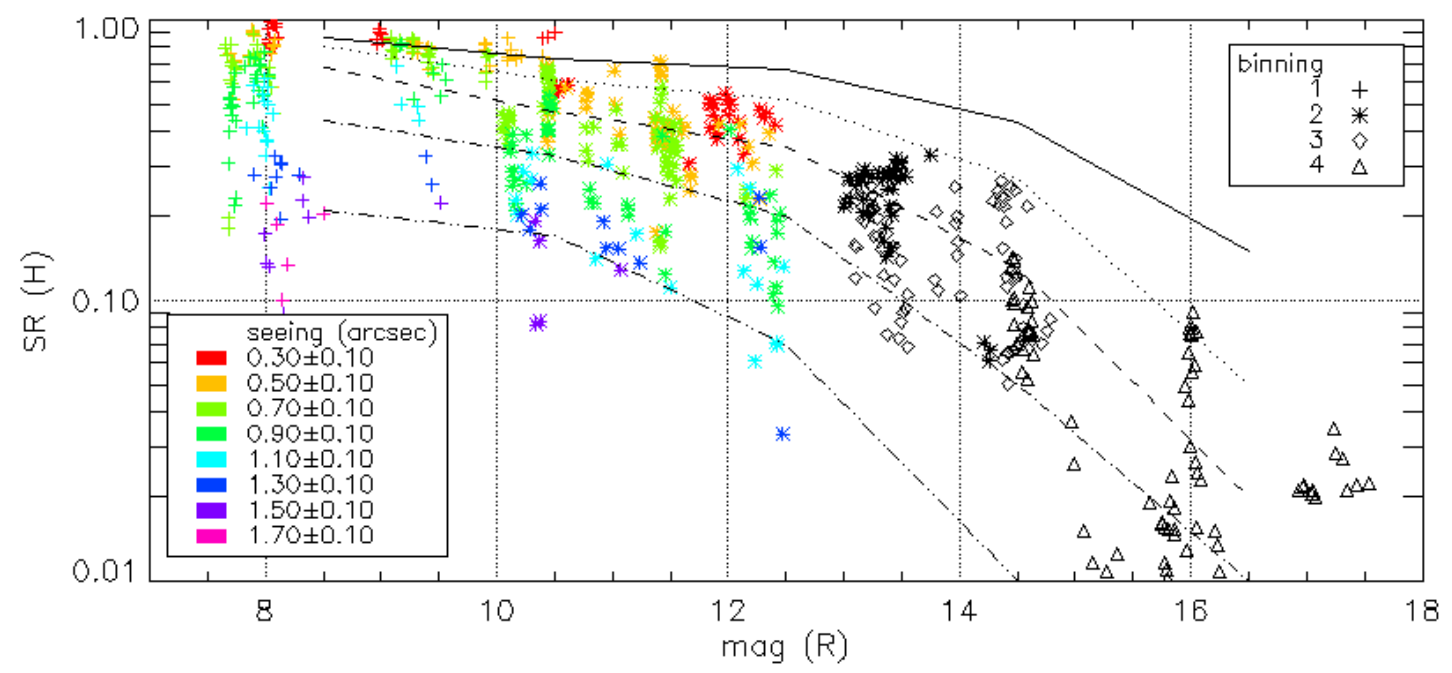

Figure 3. (Color online) Summary plot of FLAO\#1 performance commissioning results. Strehl Ratio in $\mathrm{H}$ band versus star magnitude $(\mathrm{R})$ and for different seeing values (estimated from AO real-time data). Points $\left(M_{R}>13\right)$ for which seeing values are strongly under-estimated are shown in black. Different binning modes are indicated with symbols. Lines correspond to expected performance from numerical simulations with different seeing values; from top to bottom: 0.6 ", $0.8 ", 1.0 ", 1.2 "$, and 1.5".

Figure 4 shows some examples of AO-corrected PSFs sampling the range of star magnitudes $\left(8<M_{R}<17\right)$, and for a seeing value of about $0.8 \mathrm{arcsec}$. Note that a diffraction-limited resolution of $40 \mathrm{mas}$ is achieved down to magnitude $\sim 12$. In the next subsections we will discuss some particular cases.
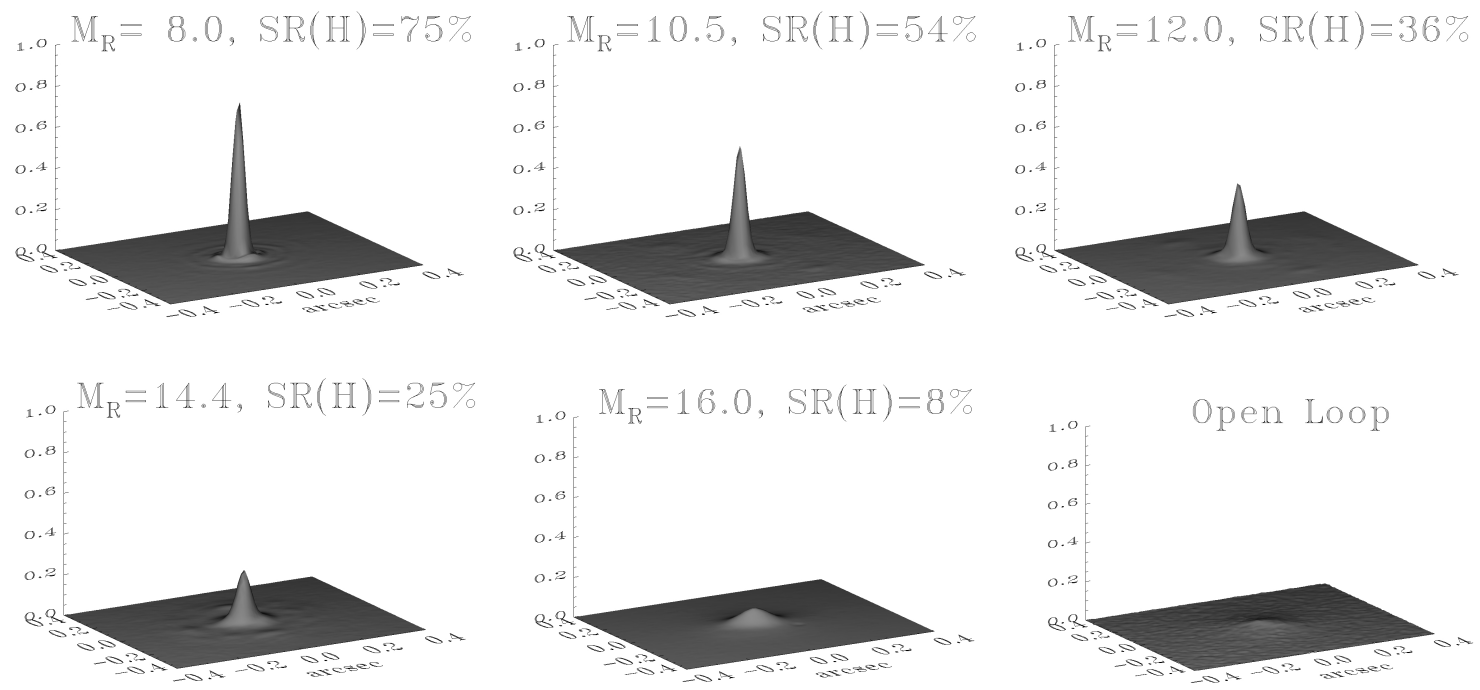

Figure 4. Examples of AO-corrected PSFs (normalized to the diffraction-limited peak) acquired at different star magnitudes.

The system configurations are listed in Table 1 . The seeing value for all of these acquisitions was about 0.8 arcsec. An OL PSF is also shown for comparison. 


\subsection{Performance at the bright end: high-contrast imaging}

Figure 5 shows an example of a high-order corrected PSF in $\mathrm{H}$ band with a SR of $>80 \%$. The equivalent R magnitude of the GS (HD175658) is 6.5 and the on-line estimated seeing oscillated between 0.6 and 0.8 arcsec. The AO loop was running at $1 \mathrm{kHz}$ controlling $400 \mathrm{KL}$ modes. Some residual tip/tilt vibrations at $\sim 13.4 \mathrm{~Hz}$ of 6 to 8 mas rms were present. Figure 5 also shows the radial-averaged profiles of the AO-corrected and the diffraction-limited PSFs. It is important to note that a contrast better than $10^{4}$ is achieved at a radial distance of $0.4 \operatorname{arcsec}$ radius from the central peak, just before the turbulence residual halo occurring at $\sim 0.47$ arcsec. This image confirms on sky for the first time the deep annular region where the non-aliased correction offered by the pyramid sensor achieves the maximum contrast ${ }^{[10]}$.
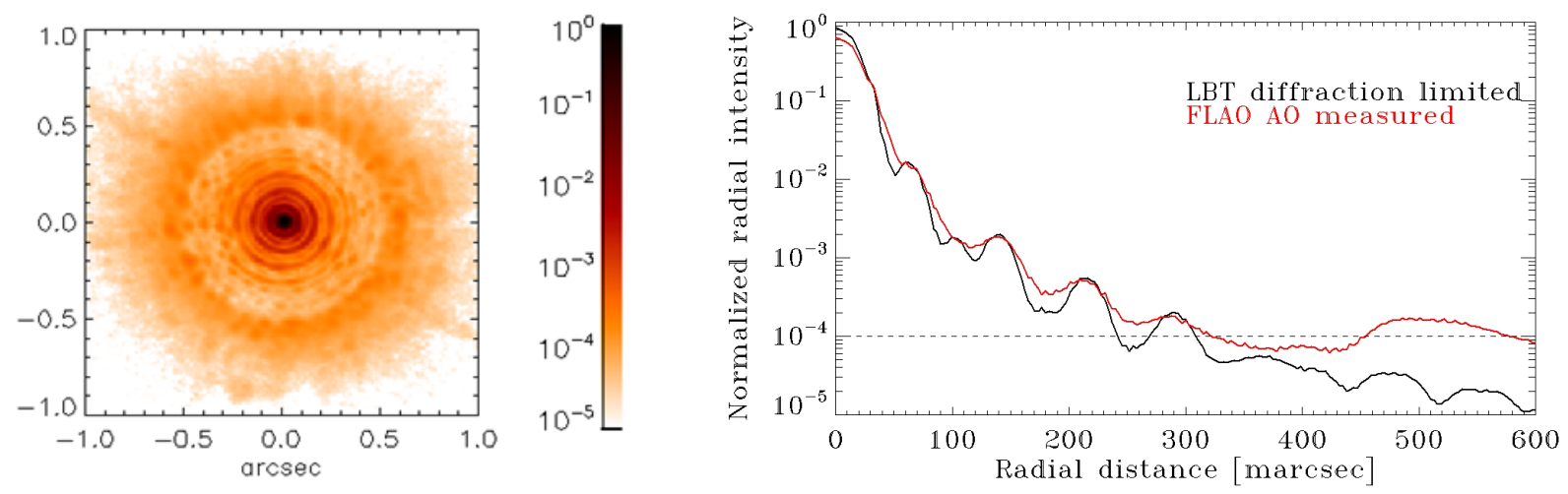

Figure 5. (Left) High-order AO-corrected PSF using a bright star $\left(M_{R}=6.5\right)$ under median seeing conditions (seeing 0.7" \pm 0.1 "). (Right) Comparison between the diffraction-limited PSF (black full curve) and the AOcorrected PSF profiles (red full curve). Profiles are normalized to the diffraction-limited peak.

\subsection{Performance at the faint end}

Let us now discuss the performance of the FLAO system with faint reference stars, close to the current limiting magnitude. Figure 6 shows an example of the results obtained at $M_{R}=17.2$. The star observed was LP154-66 $\left(M_{R}=13.20\right.$, $\left.M_{H}=10.17\right)$, and a neutral density filter was placed on the WFS channel to emulate a $17.2 \mathrm{R}$-magnitude star. The system was operated with binning mode \#4 and controlling only 10 modes giving a SR(H) of $\sim 3.5 \%$. The FWHM of the AOcorrected PSF is reduced a factor of 1.7 with respect to the seeing-limited one, and their peaks ratio equals 2.6.

A seeing estimate for this acquisition was not available from the DIMM. We can nevertheless use the FWHM of the seeing-limited PSF and, assuming a given outer scale value, estimate the seeing as in Tokovinin $(2002)^{[11]}$. Following this method, and considering an outer scale of $L_{0}=40 \mathrm{~m}$, we get a seeing estimate of 0.72 " (@ 500nm) for this observation.
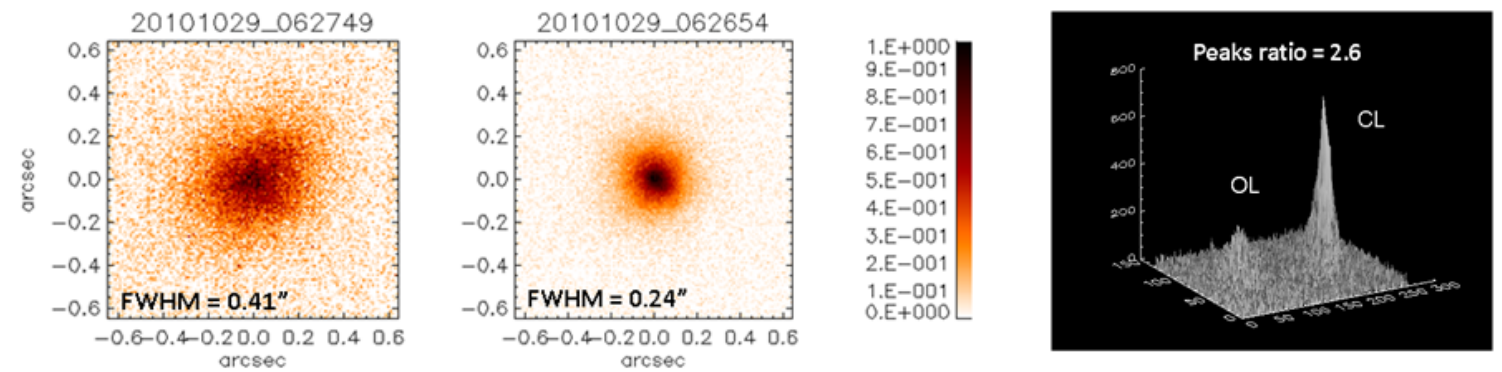

Figure 6. Example of the FLAO performance in $\mathrm{H}$ band at its faint end $\left(M_{R}=17.2\right)$. (Left) Seeing-limited image. (Middle) AO-corrected image. System parameters: binning mode \#4, 10 controlled modes, $100 \mathrm{~Hz}$, and a modulation of $\pm 6 \lambda_{s} / D$. Both images are normalized to their peak intensities. (Right) Images placed side by side to show the gain in energy concentration. These images were produced from 5 IRTC frames of $3 \mathrm{~s}$ exposure time each. 


\section{NGS AND LGS ADAPTIVE OPTICS SYSTEMS AFTER LBT RESULTS}

We discuss in this section the case of adaptive correction on a small FoV, more precisely the FoV limited by the size of the isoplanatic patch of the atmosphere. Recall that the development of Laser Guide Star (LGS) systems to create suitable reference stars for adaptive optics was mostly driven by the relative inefficiency of the first NGS-based AO systems for science applications. Indeed, the efficiency in exploiting the star flux was in the past lower, with the consequence of achieving a limited sky coverage of the order of one to a few percent, in most cases. Also, the fast readout CCDs were noisier and the efficiency at low flux levels of the wave-front sensors had to be improved.

In the case of the LBT, the quest for efficiency led to the development of its two AO key components. On one hand, the ASM allows to minimize the number of reflections giving the WFS the possibility to catch the light after only three reflections (primary mirror, ASM, and the folding tertiary); on the other hand, the modulated pyramid allows for a gain of the order of 1-2 limiting magnitudes with respect to commonly used Shack-Hartmann sensors ${ }^{[12][13]}$. In terms of system efficiency, a future upgrade consists in replacing the current WFS camera (E2V CCD 39) with a Low Light Level CCD (LLLCCD) also called Electron Multiplied CCD (EMCCD) as proposed by Carbillet and Riccardi (2010) ${ }^{[14]}$.

\subsection{Improving NGS AO performance and Sky Coverage with an LLLCCD}

We have evaluated the performance of an LBT-like system using an LLLCCD with the numerical simulator we developed for FLAO performance verification ${ }^{[7]}$. The main parameters of the LLLCCCD we simulated are the following: $0.1 \mathrm{e}^{-}$RMS noise from Clock Induced Charges (CIC), 0.03 e- RMS of RON at $1 \mathrm{kfps}$. The main atmospheric parameters used in the simulations are: seeing of 0.6 arcsec, outer scale of $L_{0}=40 \mathrm{~m}$, and a wind speed of $15 \mathrm{~m} / \mathrm{s}$.

Results of the simulations in terms of SR in K band $(2.2 \mu \mathrm{m})$ as a function of the GS magnitude are reported in Figure 7 for the FLAO system with a CCD39 and with an LLLCCD. As a comparison, we report on the same plot a typical performance curve for the LGS AO system of the Keck telescope, one of the most successful LGS-based AO systems ${ }^{[15]}$. Performance curves are reported for a seeing value of $0.6 \operatorname{arcsec}$ (median seeing in Mauna Kea ${ }^{[16]}$ ). We recall here briefly that the LGS system needs to use an NGS as the reference for the tip/tilt signal that is not provided by the LGS ${ }^{[17]}$. However, in this case the NGS is used to measure only the overall tip/tilt and so it can be fainter than the usual reference star for an NGS system. Figure 7 shows that the LGS system has a better limiting magnitude with respect to the FLAO NGS cases. For instance, for a SR of $10 \%$ in K band, we obtain 18.4 and 19.0 limiting magnitudes for NGS+LLLCCD and LGS respectively. On the other hand, the NGS+LLLCCD system performance is higher than the LGS system up to magnitude 16.8 reaching a SR higher than $70 \%$ up to magnitude 15.2. We note that one of the main limitations of LGS performance in the bright end is focus anisoplanatism error ${ }^{[18]}$.

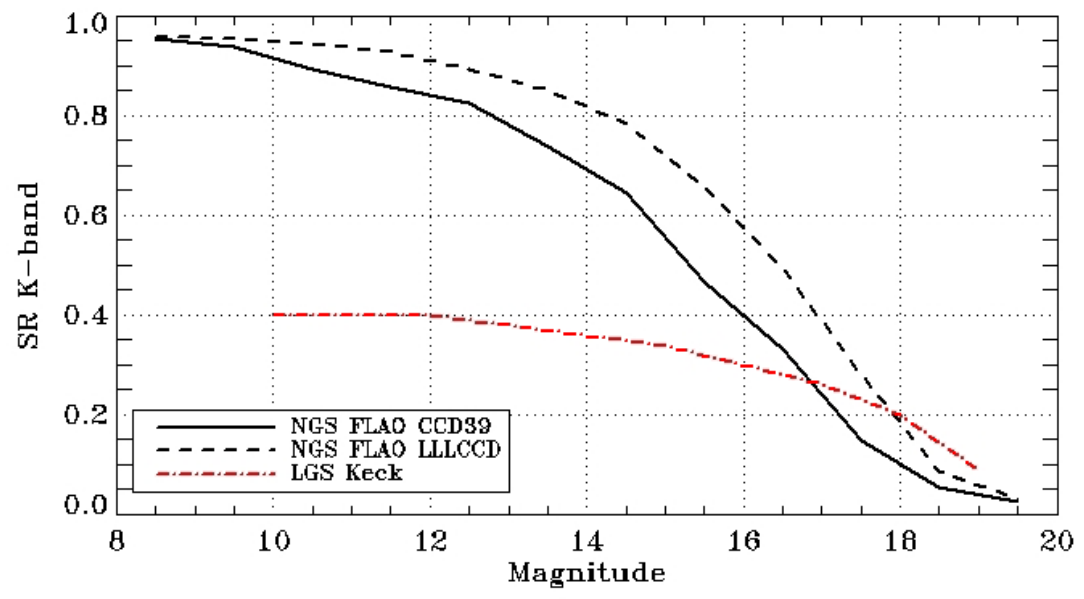

Figure 7. Simulated performance (seeing of 0.6 arcsec) in terms of SR in K band versus star magnitude for the LBT FLAO system and of the improved version using an LLLCCD. The performance of the Keck LGS AO system is shown for comparison. 


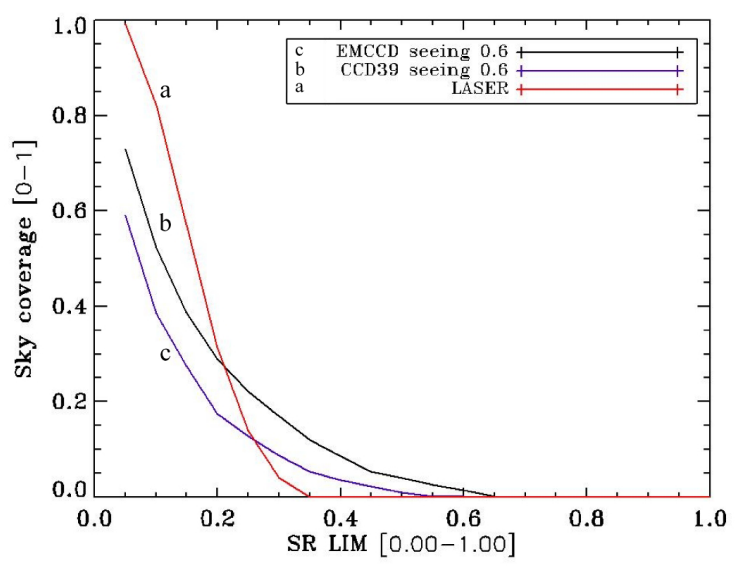

Figure 8 Sky coverage results for the Steidel \& al (2003) sample of galaxies using the actual LBT FLAO system (curve c), the improved version using LLLCCD (curve b) and the Keck AO LGS system (curve a). The analyses were made under 0.6 "seeing values.

We computed the Sky Coverage (SC) for the two systems in question (NGS+LLLCCD and LGS systems) for a seeing value of 0.6 arcsec with isoplanatic and isokinetic angles of 40 and 73 arcsec respectively in $\mathrm{K}$ band. The faint limiting magnitudes considered imply very high sky coverage at low galactic latitudes for both NGS and LGS systems and the comparison is not so interesting. We decided to compute the SC for an astronomical sample of targets located at high galactic latitude. In particular, we selected a sample of about 850 galaxies at $z=3$ from the Steidel $\operatorname{catalog}^{[19]}$ and computed the SR performance according to the plot already shown in Figure 7. The results are shown in Figure 8 where the SC achieved for a given SR is reported for both systems. As a reference, the same curve for the NGS+CCD39 system is also shown in the plot. The result here is that for a required SR of $10 \%$ in $\mathrm{K}$ band the LGS system achieves an $84 \% \mathrm{SC}$ while the NGS+LLLCCD system a 54\% SC.

On the other hand, for a required SR higher than 25\%, the NGS system has a better SC than the LGS system, being on average about $15 \%$. In summary, the above computations show that an LBT-like AO system upgraded from the FLAO system being already demonstrated on sky, has comparable performance to an LGS system as SC is concerned, and has definitely better performance when higher SR values are required.

\subsection{Future developments for AO systems}

An interesting point about NGS and LGS comparison is the use of adaptive optics systems at wavelengths shorter than the $\mathrm{K}$ and $\mathrm{H}$ bands like $\mathrm{J}$ or even visible wavelengths. Because of the focus anisoplanatism effect, the single LGS correction becomes less and less effective when going to shorter wavelengths ${ }^{[18]}$. Hence, such a single laser system for an $8 \mathrm{~m}$ class telescope is limited to correction in $\mathrm{K}$ and $\mathrm{H}$ band. This limitation does not exist for the NGS system that can be pushed to shorter wavelengths provided a sufficient number of photons and actuators exist. The LBT telescope is a first example of this, having achieved about $50 \%$ SR in J band and $20 \%$ of SR at $850 \mathrm{~nm}$. The scaling of LBT on-sky results to shorter wavelengths is shown in Figure 9. Let us mention that the 6.5 Magellan telescope ${ }^{[20]}$ is in the testing phase of an LBT-like system aimed at visible observations. A similar system could be developed for the ESO UT4 telescope being equipped in the next future with an adaptive secondary having more than 1100 actuators ${ }^{[21]}$. Such a system could deliver 15 mas FWHM at $0.6 \mu \mathrm{m}$. In the LGS case, visible AO requires to solve the focus anisoplanatism issue using more than one LGS and so in turn using more than one NGS for the measurements of wavefront low-order modes ${ }^{[22]}$. On the other hand, NGS systems do no require major changes to be operational at shorter wavelengths as already discussed.

Finally, if we move to AO systems for ELTs, the design of an LGS system becomes very complex considering the number of LGS and NGS needed, the needs to deal with the spot elongations effect, and several other issues arising from the use of LGS such as the variability of the sodium density profile, etc. On the contrary, an NGS-based system for an ELT is built exactly as a system for an $8 \mathrm{~m}$ telescope, the only difference being the number of actuators and subapertures. Such considerations summed to the easiness of operation and reduced cost of an NGS-based AO system suggests rethinking the use of LGS in AO to improve system SC and reliability for 8m and ELT class telescopes. This at least for AO systems having the corrected FoV limited by the atmospheric isoplanatic patch. 


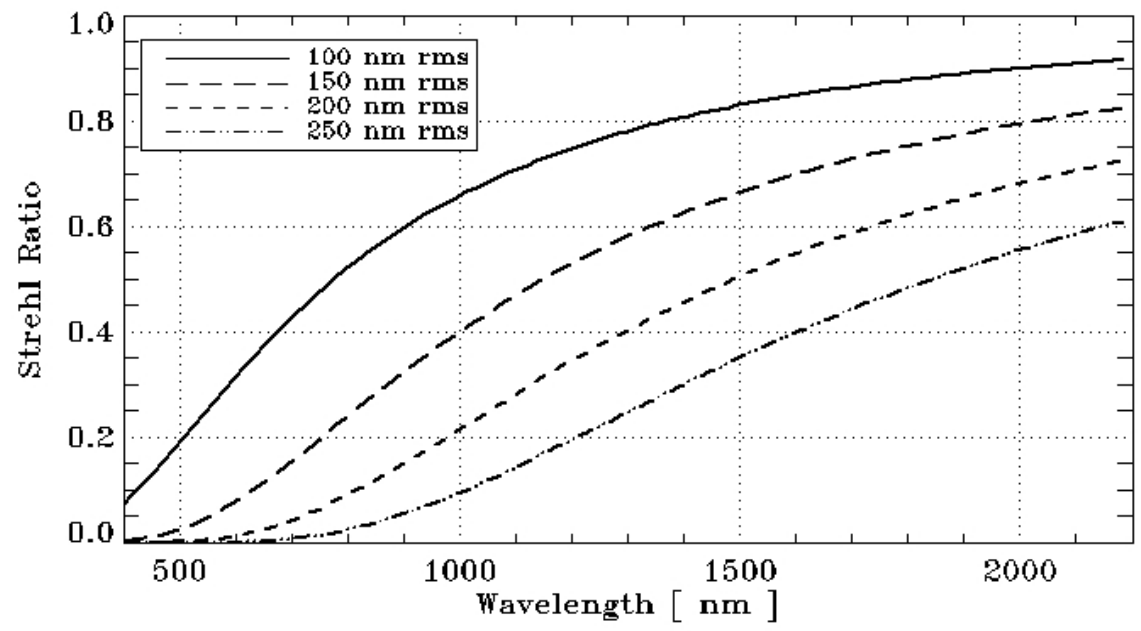

Figure 9. Strehl Ratio scaling as a function of the wavelength. Each curve represents a different level of wave-front correction corresponding to SR values in $\mathrm{H}$ band of $85 \%, 70 \%, 55 \%$, and $40 \%$.

\section{CONCLUSIONS}

The First Light AO system (FLAO\#1) of the 2x8.4m LBT telescope has been commissioned on sky in the period from June 2010 to August 2011. The achieved results — such as H band SR higher than $80 \%$, image contrast better than $10^{4}$, and loop closure down to 17.5 magnitude star - sets a new standard for ground based astronomical AO systems. At the same time these results show that adaptive secondary mirrors and pyramid sensors are mature technologies to be used in the design of future $\mathrm{AO}$ systems for $8 \mathrm{~m}$ and ELT class telescopes.

Finally, an elaboration of the LBT experience does show that an LBT-like system using an LLLCCD can achieve, in the case studied of extragalactic objects, a sky coverage of 54\% comparable to the one obtained with an LGS of $84 \%$. Moreover, a better sky coverage at SR higher than $25 \%$ can be achieved by the NGS system. These results, coupled with relevant limitations of LGS systems like cone effect and the need for low-order modes sensing with an NGS, suggest that the choice of using LGS systems instead of NGS systems to improve AO system efficiency should be revisited.

\section{ACKNOWLEDGEMENTS}

The authors would like to thank the LBTO personnel for their continuous and timely support during the integration and commissioning of the system.

\section{REFERENCES}

[1] Hill, J. M., “The Large Binocular Telescope,” App. Opt. 49(16), D115-D122 (2010).

[2] Esposito, S., Riccardi, A., Quirós-Pacheco, F., Pinna, E., Puglisi, A., Xompero, M., Briguglio, R., Busoni, L., Fini, L., Stefanini, P., Brusa, G., Tozzi, A., Ranfagni, P., Pieralli, F., Guerra, J. C., Arcidiacono, C. and Salinari, P. "Laboratory characterization and performance of the high-order adaptive optics system for the Large Binocular Telescope,” App. Opt. 49(31), G174-G189 (2010).

[3] Esposito, S., Riccardi, A., Fini, L., Puglisi, A. T., Pinna, E., Xompero, M., Briguglio, R., Quirós-Pacheco, F., Stefanini, P., Guerra, J. C., Busoni, L., Tozzi, A., Pieralli, F., Agapito, G., Brusa-Zappellini, G., Demers, R., Brynnel, J., Arcidiacono, C., and Salinari, P., "First light AO (FLAO) system for LBT: final integration, acceptance test in Europe, and preliminary on-sky commissioning results," Proc. SPIE 7736, 773609 (2010). 
[4] Seifert, W., Appenzeller, I., Baumeister, H., Bizenberger, P., Bomans, D., Dettmar, R. J., Grimm, B., Herbst, T., Hofmann, R., Juette, M., Laun, W., Lehmitz, M., Lemke, R., Lenzen, R., Mandel, H., Polsterer, K., Rohloff, R. R., Schuetze, A., Seltmann, A., Thatte, N. A., Weiser, P. and Xu, W., "LUCIFER: a multi-mode NIR instrument for the LBT," Proc. SPIE 4841, 962-973 (2003).

[5] Riccardi, A., Xompero, M., Briguglio, R., Quirós-Pacheco, F., Busoni, L., Fini, L., Puglisi, A., Esposito, S., Arcidiacono, C., Pinna, E., Ranfagni, P., Salinari, P., Brusa, G., Demers, R., Biasi, R., and Gallieni, D., "The adaptive secondary mirror for the Large Binocular Telescope: optical acceptance test and preliminary on-sky commissioning results," Proc. SPIE 7736, 77362C (2010).

[6] Ragazzoni, R., "Pupil plane wavefront sensing with an oscillating prism”, J. Mod. Opt. 43(2), 289-293 (1996).

[7] Quirós-Pacheco, F., Busoni, L., Agapito, G., Esposito, S., Pinna, E., Puglisi, A., and Riccardi, A., "First light AO (FLAO) system for LBT: performance analysis and optimization," Proc. SPIE 7736, 77363H (2010).

[8] Fusco, T., Rousset, G., Rabaud, D., Gendron, E., Mouillet, D., Lacombe, F., Zins, G., Madec, P. Y., Lagrange, A. M., Charton, J., Rouan, D., Hubin, N., and Ageorges, N., "NAOS on-line characterization of turbulence parameters and adaptive optics performance,” J. Opt. A: Pure Appl. Opt. 6, 585-596 (2004).

[9] Foppiani, I., Hill, J. M., Lombini, M., Bregoli, G., Cosentino, G., Diolaiti, E., Herbst, T. M., Innocenti, G., Meschke, D., Miller, D. L., Rohloff, R. R., and Schreiber, L., "An instrument for commissioning the active and adaptive optics of modern telescopes: the Infrared Test Camera for the Large Binocular Telescope," Exp. Astron. (2011), doi: 10.1007/s10686-011-9229-2

[10] Verinaud, C., "On the nature of the measurements provided by a pyramid wave-front sensor", Opt. Comm. 233, 27-38 (2004).

[11] Tokovinin, A., "From differential image motion to seeing”, PASP 114, 1156-1166 (2002).

[12] Ragazzoni, R., Farinato, J., "Sensitivity of a pyramidic wavefront sensor in closed loop adaptive optics", A\&A 350, L23-L26 (1999).

[13] Esposito, S., and Riccardi, A., "Pyramid Wavefront Sensor behavior in partial correction Adaptive Optic systems", A\&A 369, L9-L12 (2001).

[14] Carbillet, M., and Riccardi, A., "Low-light-level charge-coupled devices for pyramid wavefront sensing on $8 \mathrm{~m}$ class telescopes: what actual gain?”, App. Opt. 49(31), G167-G173 (2010).

[15] van Dam, M. A., Bouchez, A. H., Le Mignant, D., Johansson, E. M., Wizinowich, P. L., Campbell, R. D., Chin J., Hartman, S., K., Lafon, R. E., Stomski, J. Jr., and Summers, D. M., “The W. M. Keck Observatory Laser Guide Star Adaptive Optics System: Performance Characterization”, PASP 118, 310-318 (2006).

[16] Tokovinin, A., Vernin, J., Ziad, A., Chun, M., “Optical Turbulence Profiles at Mauna Kea Measured by MASS and SCIDAR", PASP 117, 395-400 (2005).

[17] Fried, D. L., “Artificial-guide-star tilt-anisoplanatism: its magnitude and (limited) amelioration”, OSA Technical Digest Series 23, 363-369 (1995).

[18] Sandler, D. G., Stahl, S., Angel, J. R. P., Lloyd-Hart, M. and McCarthy, D., “Adaptive optics for diffractionlimited infrared imaging with 8-m telescopes", J. Opt. Soc. Am. A 11(2), 925-945 (1994).

[19] Steidel, C. C., Adelberger, K. L., Shapley, A. E., Pettini, M., Dickinson, M. And Giavalisco, M., "Lyman Break Galaxies at Redshift z 3: Survey Description and Full Data Set”, Astrophys. J. 592(2), 728-754 (2003).

[20] Close, L. M., Gasho, V., Kopon, D., Males, J., Follette, K. B. and Brutlag, K., "The Magellan Telescope Adaptive Secondary AO System: a visible and mid-IR AO facility”, Proc. SPIE 7736, 773605 (2010).

[21] Arsenault, R., Biasi, R., Gallieni, D., Riccardi, A., Lazzarini, P., Hubin, N., Fedrigo, E., Donaldson, R., Oberti, S., Stroebele, S., Conzelmann, R. and Duchateau, M. "A deformable secondary mirror for the VLT”, Proc. SPIE 6272, 62720V (2006).

[22] Tallon, M. and Foy, R., “Adaptive Telescope with Laser Probe - Isoplanatism and Cone Effect”, A\&A 235(12), 549-557 (1990). 